\title{
Explicit coupling of acoustic and elastic wave propagation in finite difference simulations
}

\author{
Longfei Gao* and David Keyes ${ }^{\dagger}$
}

\begin{abstract}
We present a mechanism to explicitly couple the finite-difference discretizations of 2D acoustic and isotropic elastic wave systems that are separated by straight interfaces. Such coupled simulations allow the application of the elastic model to geological regions that are of special interest for seismic exploration studies (e.g., the areas surrounding salt bodies), while with the computationally more tractable acoustic model still being applied in the background regions. Specifically, the acoustic wave system is expressed in terms of velocity and pressure while the elastic wave system is expressed in terms of velocity and stress. Both systems are posed in first-order forms and discretized on staggered grids. Special variants of the standard finite-difference operators, namely, operators that possess the summation-by-parts property, are used for the approximation of spatial derivatives. Penalty terms, which are also referred to as the simultaneous approximation terms, are designed to weakly impose the elastic-acoustic interface conditions in the finite-difference discretizations and couple the elastic and acoustic wave simulations together. With the presented mechanism, we are able to perform the coupled elastic-acoustic wave simulations stably and accurately. Moreover, it is shown that the energy-conserving property in the continuous systems can be preserved in the discretization with carefully designed penalty terms.
\end{abstract}

\section{INTRODUCTION}

Waves propagating through earth media are routinely employed in seismic exploration. Many modern seismic imaging techniques require simulating seismic wave propagations repeatedly for numerous source terms and various instances of media parameters, cf., for instance, Symes (2009), Virieux and Operto (2009), Schuster (2014), and the references therein.

Since the true earth media are inaccessible to us, acoustic and elastic model assumptions are commonly used as surrogates in practice. More complex model assumptions exist, such as Biot's theory for porous media (Biot, 1956a b), which can in theory provide more comprehensive depiction of the seismic waves, but is difficult to utilize effectively in current practice due to its expensive simulation cost and expanded number of model parameters. Between the acoustic and elastic media assumptions, the latter is considered more accurate for earth media since, in addition to the compressional wave supported by the former, it also supports shear and surface waves, which are commonly observed in seismic survey data.

However, the simulation cost associated with the elastic model is significantly higher than that of the acoustic model. Consider the following simple thought experiment involving a homogeneous medium with compressional wave-speed $c_{p}$ and shear wave-speed $c_{s}$, where 
$c_{p}>c_{s}$. When simulating seismic waves, the spatial grid spacing is usually decided on a points per minimal wavelength basis. For a given frequency $f$, the minimal wavelength in elastic simulation (denoted as $\lambda_{\text {min }}^{E}$ ) is determined by the smaller shear wave-speed as follows

$$
\lambda_{\min }^{E}=\frac{c_{s}}{f}
$$

the minimal wavelength in acoustic simulation (denoted as $\lambda_{\min }^{A}$ ) is determined by the compressional wave-speed as follows

$$
\lambda_{\min }^{A}=\frac{c_{p}}{f},
$$

since the acoustic simulation concerns only the compressional wave. For a prescribed number of grid points per minimal wavelength (denoted as $N_{\mathrm{ppw}}$ hereafter), the resulting spatial grid spacings in elastic and acoustic simulations are

$$
\Delta_{x}^{E}=\frac{\lambda_{\mathrm{min}}^{E}}{N_{\mathrm{ppw}}}=\frac{c_{s}}{f \cdot N_{\mathrm{ppw}}} \quad \text { and } \quad \Delta_{x}^{A}=\frac{\lambda_{\mathrm{min}}^{A}}{N_{\mathrm{ppw}}}=\frac{c_{p}}{f \cdot N_{\mathrm{ppw}}},
$$

respectively, which leads to the ratio $\Delta_{x}^{A} / \Delta_{x}^{E}=c_{p} / c_{s}$. Furthermore, since seismic wave simulations usually employ explicit time stepping methods, the time step lengths are usually dictated by the Courant-Friedrichs-Lewy (CFL) stability condition. In short, we have

$$
\Delta_{t}^{E} \propto \frac{\Delta_{x}^{E}}{c_{p}} \quad \text { and } \quad \Delta_{t}^{A} \propto \frac{\Delta_{x}^{A}}{c_{p}}
$$

where $\Delta_{t}^{E}$ and $\Delta_{t}^{A}$ stand for the time step lengths in elastic and acoustic simulations, respectively. Assuming that the Courant numbers for both cases are the same, we then have the ratio $\Delta_{t}^{A} / \Delta_{t}^{E}=\Delta_{x}^{A} / \Delta_{x}^{E}=c_{p} / c_{s}$.

Taking the ratio $c_{p} / c_{s}$ to be $2,{ }^{\ddagger}$ then, with three spatial dimensions and one temporal dimension, the overall space-time discretization points in the elastic simulation can be 16 times as many as that of the acoustic simulation. Furthermore, when posed as first-order systems in terms of velocity and pressure (acoustic case) or velocity and stress (elastic case), the spatial derivatives appearing in the isotropic elastic system are three times as many as that of the acoustic system; approximation of these spatial derivatives (e.g., via applying stencils) represents majority of the computations during seismic wave simulations. Altogether, the ratio of computational costs associated with these two models reaches almost 50 in this simple thought experiment.

The above observation motivates this study on coupling elastic and acoustic models for seismic wave simulations so that application of the more expensive elastic model can be restricted to regions where it makes significant impacts. One such region is the area surrounding a salt body, which is of particular interest in seismic exploration since salt bodies often form caps for oil and gas reservoirs. The contrast in wave-speed values between a salt body and its surrounding sediments can be significant. For instance, the salt body can have compressional wave-speed values above $4500 \mathrm{~m} / \mathrm{s}$ and shear wave-speed values above $2500 \mathrm{~m} / \mathrm{s}$ with its surrounding sediments having compressional wave-speed values around

\footnotetext{
${ }^{\ddagger}$ The ratio $c_{p} / c_{s}=2$ is common for fluid saturated sediments, cf., for instance, Gregory (1976). Moreover, according to Hamilton (1979), exceptionally high $c_{p} / c_{s}$ ratios (above 13) can appear in sea floor sediments.
} 
2000 m/s (cf. Bourbié et al., 1987; Leveille et al., 2011; Jackson and Hudec, 2017). This high contrast in wave-speeds often leads to strong wave conversions at salt boundaries, which can be problematic for seismic imaging and interpretation under the acoustic assumption (cf. Ogilvie and Purnell, 1996; Lu et al., 2003, Jones and Davison, 2014), among others.

Specifically, when entering the salt body, the downward propagating $\mathrm{P}$ wave can split into transmitted $\mathrm{P}$ and $\mathrm{S}$ waves at the top of salt and continue their paths inside the salt body. Upon reflection at the base of salt, both transmitted waves can return upward as $\mathrm{P}$ or $\mathrm{S}$ waves. When exiting the salt body, the base-of-salt reflected upward propagating $\mathrm{S}$ wave can convert to $\mathrm{P}$ wave again at the top of salt. In total, four possible wave paths exist for waves entering and exiting the salt body as $\mathrm{P}$ wave, denoted as PPPP, PPSP, PSPP, and PSSP following the nomenclature from Ogilvie and Purnell $(1996)^{\S}$.

In the presence of salt, all four wave paths mentioned above can carry significant energy (see Fig. 7 of Ogilvie and Purnell, 1996 and Fig. 17 of Lu et al., 2003). If migrated with the $\mathrm{P}$ wave velocity, the three wave paths containing converted waves (i.e., PPSP, PSPP, and PSSP) can obscure or misinform the sub-salt structures. On the other hand, they have the potential to be used constructively to corroborate or complement the base-of-salt image formed by the PPPP events alone. Elastic modeling in the region surrounding the salt body is crucial to correctly capture these converted waves and to facilitate the appropriate subsequent processing steps, may it be identifying and removing, or constructively exploiting these converted wave information.

A simple approach to couple the elastic and acoustic wave simulations is to use the elastic wave equation everywhere and simply set the shear wave-speed outside the interested region to zero. There are several drawbacks with this approach. First, it leads to unnecessary computations outside the interested region. Second, it introduces abrupt jumps in the shear wave-speed, which often requires finer discretization to resolve (cf. Brown, 1984; Symes and Vdovina, 2009). Third, certain solution components, such as the particle velocity tangential to the interface, are assumed to be continuous across the interface while the physical interface conditions do not pose such constraint (cf. Singh et al., 2019), which is similar to the situation in fault modeling (cf. Rojas et al., 2008). In this study, we present a mechanism to explicitly couple 2D acoustic and isotropic elastic wave simulations that are separated by straight interfaces. Geometric configuration of the abstracted simulation domain can be found in Figure 1a. Although we only address the 2D case in this study, the same methodology can be extended to the $3 \mathrm{D}$ case, since the presented interface treatment only requires modifications along the perpendicular directions of the interfaces.

Earlier works on explicitly coupling elastic and acoustic wave simulations can be found in, e.g., Komatitsch et al. (2000), Chaljub et al. (2003), Käser and Dumbser (2008), Michler et al. (2009), Wilcox et al. (2010), Rodríguez-Rozas and Diaz (2016), Ye et al. (2016), Appelö and Wang (2019), Terrana et al. (2018), which employ the finite element discretization approach. On the other hand, within the finite-difference discretization approach, some earlier works on explicitly coupling elastic and acoustic wave simulations can be found in Stephen (1983) and Stephen et al. (1985), where the elastic-acoustic interface conditions are used to set up linear systems to deduce the formulas for solution updates at the interfaces.

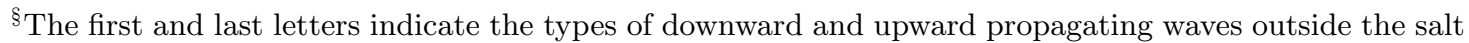
body; the two letters in the middle indicate the types of downward and upward propagating waves inside the salt body. In conventional seismic imaging practice, only $\mathrm{P}$ wave events are exploited, hence the four considered wave paths all start and end with the letter ' $\mathrm{P}$ '.
} 
Some recent works can be found in Singh et al. (2019) and Qu et al. (2020), which resort to the mimetic finite-difference approach. Interested readers may consult Rojas et al. (2008), Castillo and Miranda (2013), de la Puente et al. (2014), Shragge and Tapley (2017) and the references therein for more information about the mimetic discretization technique.

Due to its simplicity and cost-effectiveness, the finite-difference discretization approach remains the popular choice in seismic exploration, with strong interest in furthering its development. In this study, we adopt the so-called SBP-SAT technique for spatial discretization, where SBP stands for summation-by-parts and refers to the property of the finite-difference operators while SAT stands for simultaneous approximation term, which is the penalty term introduced to address boundary or interface conditions. These concepts date back to Kreiss and Scherer (1974) and Carpenter et al. (1994), respectively. Interested readers may consult the two review papers Del Rey Fernández et al. (2014) and Svärd and Nordström (2014), as well as the references therein for more details. Other more recent related works include Kozdon and Dunham (2014), Lotto and Dunham (2015), O'Reilly et al. (2017), Banks et al. (2018), Petersson and Sjögreen (2018), Gao and Keyes (2019).

In terms of the procedure, the respective wave systems on acoustic and elastic regions are first discretized using SBP finite-difference operators without concern for the interface conditions. These semi-discretized wave systems are then coupled together through penalty terms, i.e., SATs, that impose the interface conditions weakly. With carefully designed penalty terms, we show that the overall semi-discretization preserves the energy-conserving property in the continuous systems. We believe that this particular discretization approach using SBP finite-difference operators and weak imposition of the interface conditions with the energy-conserving property built in has not been presented for the coupled simulation of elastic-acoustic wave system yet and is worth sharing with the seismic community.

The rest of this paper is organized as follows. We first briefly describe the geometric configuration of the simulation domain and the acoustic and isotropic elastic wave systems under consideration. We then present the semi-discretizations on both acoustic and elastic regions using SBP finite-difference operators, respectively, as well as the penalty terms that couple them together. Finally, we demonstrate the efficacy of the proposed interface treatment with numerical examples.

\section{PROBLEM DESCRIPTION}

Figure 1aillustrates the abstracted geometric configuration under consideration, where a rectangular target region is enclosed in the background region. An isotropic elastic medium is considered for the target region while an acoustic medium is considered for the background region. The respective equation systems describing the wave propagation in these two regions are presented in the subsequent sections.

For spatial discretization, we adopt the staggered grids finite-difference approach that dates back to Yee (1966) and has enjoyed its popularity in seismic exploration studies, cf. Madariaga (1976), Virieux (1984), Virieux (1986), Levander (1988), among others. The staggered discretization grids are illustrated in Figure 1b. Interfaces between the two regions are duplicated and are included in the discretizations of both sides. 


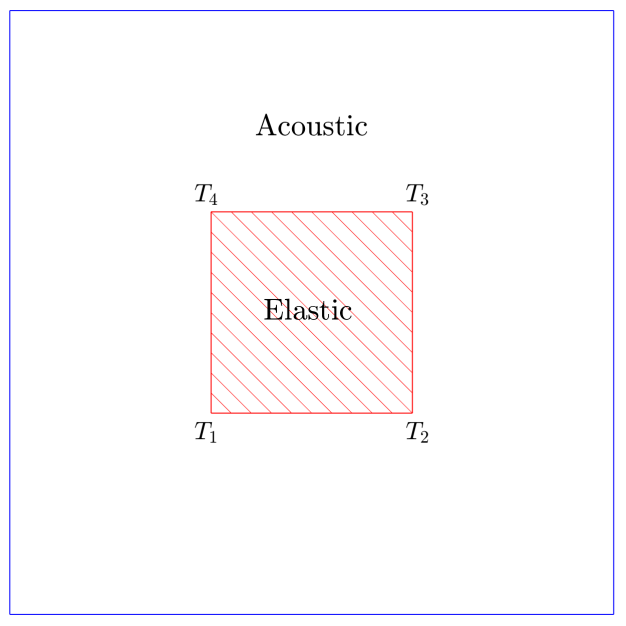

a)

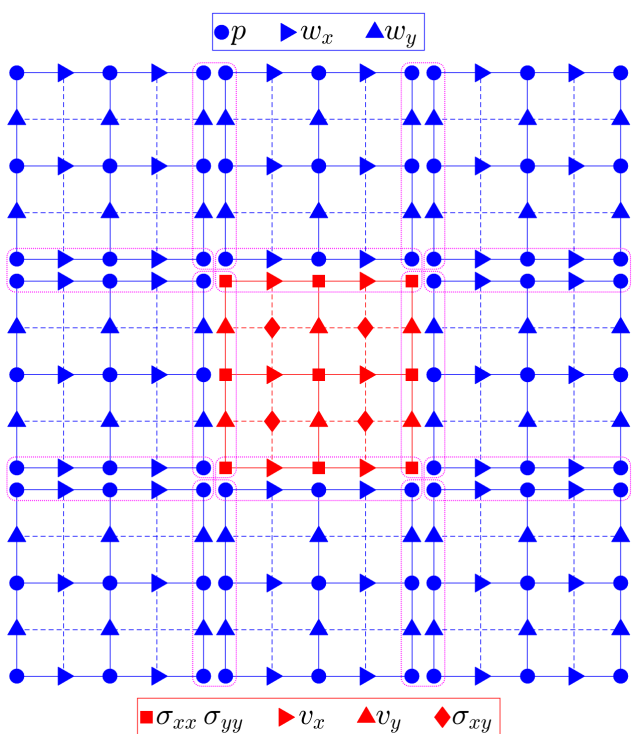

b)

Figure 1: Illustration of the geometric configuration of the simulation domain and the associated discretization grids. (a) The target (elastic) region is enclosed in the background (acoustic) region; (b) Staggered grids are used to discretize the wave systems in both regions. Interfaces are encircled by the magenta lines.

We note here that in Figure $1 \mathrm{~b}$, the entire 2D simulation domain is divided into nine rectangular blocks. Artificial interfaces are drawn between adjacent acoustic blocks as well. These artificial interfaces are also addressed using the SBP-SAT technique. Interested readers may consult Gao et al. (2019) for the SATs associated with these artificial interfaces. Discretizations over the entire simulation domain are then addressed in a block-wise manner, which eases the upcoming discussion. The actual implementation is also organized in such a block-wise manner, which modularizes the code nicely.

\section{Acoustic background region}

For the background region, i.e., the entire simulation domain except the target region as illustrated in Figure 1a, the wave propagation is described by the $2 \mathrm{D}$ acoustic wave system posed in the following first-order form:

$$
\left\{\begin{aligned}
\rho \frac{\partial w_{x}}{\partial t} & =\frac{\partial p}{\partial x} \\
\rho \frac{\partial w_{y}}{\partial t} & =\frac{\partial p}{\partial y} \\
\frac{1}{\rho c^{2}} \frac{\partial p}{\partial t} & =\frac{\partial w_{x}}{\partial x}+\frac{\partial w_{y}}{\partial y}
\end{aligned}\right.
$$

where physical parameters $\rho$ and $c$ represent density and compressional wave-speed, respectively; solution variables $w_{x}, w_{y}$, and $p$ represent horizontal particle velocity, vertical particle velocity, and the negative of pressure, respectively. The choice of expressing the 
acoustic wave equation in terms of the negative of pressure, as opposed to pressure itself, improves the consistency between the above acoustic wave system and the elastic wave system presented later, which in turn improves the clarity and consistency of the interface treatment presented below.

The kinetic and potential energy density functions associated with equation 5 are defined as

$$
\varrho_{k}^{A}=\frac{1}{2} \rho w_{i} w_{i} \quad \text { and } \quad \varrho_{p}^{A}=\frac{1}{2} \frac{1}{\rho c^{2}} p^{2}
$$

respectively, where the Einstein summation convention applies to the repeated subscript index $i$. The total continuous energy associated with equation 5 is then defined as

$$
e^{A}=\int_{\Omega_{B}} \frac{1}{2} \rho w_{i} w_{i} d_{\Omega_{B}}+\int_{\Omega_{B}} \frac{1}{2} \frac{1}{\rho c^{2}} p^{2} d_{\Omega_{B}}
$$

where $\Omega_{B}$ stands for the background region. Differentiating equation 7 with respect to time, substituting in equations $5 \mathrm{a}-5 \mathrm{c}$, and then applying the divergence theorem, we arrive at

$$
\frac{d e^{A}}{d t}=\int_{\partial \Omega_{B}} p w_{i} n_{i} d_{\partial \Omega_{B}} .
$$

In equation 8, the Einstein summation convention applies to subscript index $i ; n_{i}$ represents the $i$ th component of the outward normal direction vector; $\partial \Omega_{B}$ denotes the boundaries of $\Omega_{B}$. We note here that $\partial \Omega_{B}$ includes both the exterior boundaries and the interfaces shared with the target region. Assuming that free-surface boundary condition, i.e., $p=0$, is associated with the exterior boundaries, we have that $\frac{d e^{A}}{d t}$ in equation 8 reduces to the interfaces only.

\section{Elastic target region}

For the target region illustrated in Figure 1a, the wave propagation is described by the $2 \mathrm{D}$ isotropic elastic wave system posed in the following first-order form:

$$
\left\{\begin{aligned}
\rho \frac{\partial v_{x}}{\partial t} & =\frac{\partial \sigma_{x x}}{\partial x}+\frac{\partial \sigma_{x y}}{\partial y} ; \\
\rho \frac{\partial v_{y}}{\partial t} & =\frac{\partial \sigma_{x y}}{\partial x}+\frac{\partial \sigma_{y y}}{\partial y} ; \\
s_{x x k l} \frac{\partial \sigma_{k l}}{\partial t} & =\frac{\partial v_{x}}{\partial x} ; \\
s_{x y k l} \frac{\partial \sigma_{k l}}{\partial t} & =\frac{1}{2}\left(\frac{\partial v_{y}}{\partial x}+\frac{\partial v_{x}}{\partial y}\right) ; \\
s_{y y k l} \frac{\partial \sigma_{k l}}{\partial t} & =\frac{\partial v_{y}}{\partial y},
\end{aligned}\right.
$$

where solution variables $v_{x}$ and $v_{y}$ represent horizontal and vertical particle velocities, respectively, while $\sigma_{x x}, \sigma_{x y}$, and $\sigma_{y y}$ are components of the stress tensor. Physical parameters $s_{x x k l}, s_{x y k l}$ and $s_{y y k l}$ are components of the compliance tensor, which can be expressed in terms of the Lamé parameters $\lambda$ and $\mu$ for isotropic media. However, their exact expressions are not needed for the upcoming discussion, and are omitted here. In equations $9 \mathrm{c}-9 \mathrm{e}$, the Einstein summation convention applies to subscript indices $k$ and $l$. 
The kinetic and potential energy density functions associated with equation 9 are defined as

$$
\varrho_{k}^{E}=\frac{1}{2} \rho v_{i} v_{i} \quad \text { and } \quad \varrho_{p}^{E}=\frac{1}{2} \sigma_{i j} s_{i j k l} \sigma_{k l},
$$

respectively, where the Einstein summation convention applies to subscript indices $i, j, k$ and $l$. The total continuous energy associated with equation 9 is then defined as

$$
e^{E}=\int_{\Omega_{T}} \frac{1}{2} \rho v_{i} v_{i} d_{\Omega_{T}}+\int_{\Omega_{T}} \frac{1}{2} \sigma_{i j} s_{i j k l} \sigma_{k l} d_{\Omega_{T}},
$$

where $\Omega_{T}$ stands for the target region. Similar to the acoustic case, after differentiating equation 11 with respect to time, substituting in equations $9 \mathrm{a}-9 \mathrm{e}$, and then applying the divergence theorem, we arrive at

$$
\frac{d e^{E}}{d t}=\int_{\partial \Omega_{T}} v_{i} \sigma_{i j} n_{j} d_{\partial \Omega_{T}} .
$$

In equation 12, the Einstein summation convention applies to subscript indices $i$ and $j$; $n_{j}$ represents the $j$ th component of the outward normal direction vector; $\partial \Omega_{T}$ denotes the boundaries of $\Omega_{T}$, i.e., the interfaces. We observe from equation 12 that $\frac{d e^{E}}{d t}$ reduces to the interfaces only.

\section{A remark on the forms of the wave systems}

We note here that in both the acoustic wave system (equations 5) and the elastic wave system (equations 9), the physical parameters (i.e., $\rho, c$, and $s_{i j k l}$ ) appear on the left hand side together with the temporal derivatives, in contrast to their equivalent forms more commonly used in the literature (cf. equations A-1 and A-2), where the physical parameters appear on the right hand side together with the spatial derivatives.

The forms in equations 5 and 9 are easy to maneuver in energy analysis since these physical parameters appear explicitly in the energy definitions (equations 7 and 11 ). Following the differentiation of the energy, substitution of the wave equations becomes straightforward with equations 5 and 9. Afterward, the physical parameters no longer appear in the subsequent derivations. This benefit of convenience is particularly valuable when deriving the modified discretized systems (equations 24 and 25) that incorporate the interface conditions, since one can operate on the right hand side of the systems freely without concern for the physical parameters.

However, these forms are not convenient for implementation, particularly the elastic wave system (equations 9), where the stress components are tangled together by the compliance tensor. Their equivalent forms in equations A-1 and A-2 are more suited for this purpose. Fortunately, as explained in Appendix A, the interface treatment derived based on the systems in equations 5 and 9 can be easily translated to their equivalent forms by viewing the appended penalty terms as modifications to the corresponding spatial derivative approximations. Usage of the less common forms presented above is merely a mathematical device to ease the effort for derivation and poses no hindrance for a standard and efficient implementation. 


\section{SPATIAL DISCRETIZATION}

To discretize the spatial derivatives appearing in equations 5 and 9 , we use the SBP finite-difference operators, which are variants of the standard finite-difference operators with special adaptations to boundaries or interfaces so that they mimic the integration-byparts property of the corresponding continuous operators. For acoustic and elastic wave systems, usage of such SBP operators leads to well-defined discrete energies that mimic the behaviors of the continuous energies as illustrated in equations 8 and 12 , i.e., time derivatives of the continuous energies reduce to the domain boundaries only.

Moreover, SBP finite-difference operators themselves usually do not concern boundary or interface conditions, which are typically addressed by appending penalty terms, i.e., SATs, to the semi-discretized systems. When combined with properly designed SATs, the SBP-SAT technique can deliver stable and accurate semi-discretizations with mathematical guarantee. For an illustration of the SBP-SAT technique applied on staggered grids for seismic wave problems, interested readers may consult Gao et al. (2019) and O'Reilly et al. (2017). A supplementary material is also provided to demonstrate these concepts in the context related to this article.

Previous works on wave propagation using the SBP-SAT technique can be found in Appelö and Petersson (2009), Petersson and Sjögreen (2015), Wang et al. (2016), O'Reilly et al. (2017), Gao et al. (2019), among others. For this study, we adopt the staggered grid SBP finite-difference operators presented in Gao et al. (2019), which are included in Appendix B to make this work self-contained. However, the interface treatment presented here can be easily adjusted for other choices of SBP operators. The focus of this work is rather on the proper penalty terms, i.e., SATs, that couple the elastic and acoustic discretizations together.

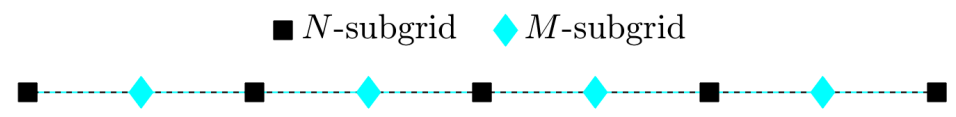

Figure 2: Illustration of the 1D subgrids. The $N$-subgrid aligns with both boundaries; the $M$-subgrid is staggered for half the grid spacing from the $N$-subgrid and has one less grid point.

Specifically, the 1D SBP operators used here are associated with the staggered grids illustrated in Figure 2, which include the 1D SBP finite-difference operators, denoted as $\mathcal{D}^{N}$ and $\mathcal{D}^{M}$, and their associated 1D norm matrices, denoted as $\mathcal{A}^{M}$ and $\mathcal{A}^{N}$, respectively. Their exact forms can be found in equation B-1. Superscripts ${ }^{N}$ and ${ }^{M}$ are appended to indicate the subgrids on which these operators act. For example, $\mathcal{D}^{N}$ can be applied to a discrete vector defined on the $N$-subgrid. The 2D SBP operators introduced later are constructed from these 1D SBP operators via tensor product.

In the interior, $\mathcal{D}^{N}$ and $\mathcal{D}^{M}$ are identical to the finite-difference operators presented in Levander (1988) and are characterized by the fourth-order stencil $[1 / 24,-9 / 8,9 / 8,-1 / 24] / \Delta x$. Near the boundaries, the stencils in $\mathcal{D}^{N}$ and $\mathcal{D}^{M}$ are adapted as illustrated in equations B-1a and B-1b, respectively, whose approximation order reduces to the second order. The adapted boundary region includes four $N$-subgrid points and three $M$-subgrid points.

The associated norm matrices enter the definitions of the discrete physical energies as the counterparts of the integrals from the continuous energies. Interested readers may 
refer to Hicken and Zingg (2013) for more information on the connection between norm matrices and quadrature rules. Although these norm matrices will not show up explicitly in implementation, they will play a pivotal role in deriving the proper interface treatment.

In this study, we limit our discussion to the case of diagonal norm matrices, i.e., $\mathcal{A}^{M}$ and $\mathcal{A}^{N}$ are diagonal, and so are the $2 \mathrm{D}$ norm matrices constructed from them as shown later in equations $\mathrm{C}-1 \mathrm{a}$ and $\mathrm{C}-3 \mathrm{a}$. Moreover, the coefficient matrices, e.g., $\boldsymbol{\rho}^{W_{x}}$ in equation 14a, are also diagonal with the finite-difference discretization approach. Consequently, the two sets of matrices are symmetric and commutable, provided that the matrix sizes are the same, which makes equations 15 and 20 proper definitions for discrete energies.

By design, the aforementioned 1D operators satisfy the following SBP property

$$
\mathcal{A}^{N} \mathcal{D}^{M}+\left(\mathcal{A}^{M} \mathcal{D}^{N}\right)^{T}=\mathcal{E}^{R}\left(\mathcal{P}^{R}\right)^{T}-\mathcal{E}^{L}\left(\mathcal{P}^{L}\right)^{T},
$$

where $\mathcal{E}^{L}$ and $\mathcal{E}^{R}$ are the canonical basis vectors that select the values of the solution vectors defined on the $N$-subgrid at the left and right endpoints, respectively; $\mathcal{P}^{L}$ and $\mathcal{P}^{R}$ are projection vectors that extrapolate the values of the solution vectors defined on the $M$-subgrid to the left and right endpoints, respectively. The exact forms of $\mathcal{E}^{L}, \mathcal{E}^{R}, \mathcal{P}^{L}$, and $\mathcal{P}^{R}$ are included in equation $\mathrm{B}-3$.

With the notations and definitions introduced above, we are now ready to discuss the SBP-SAT discretization on the 2D simulation domain. For the upcoming discussion, we attach subscripts $x$ and $y$ to the aforementioned 1D SBP operators to indicate the directions that they are associated with.

\section{Acoustic background region}

Neglecting boundaries and interfaces for now, by replacing the spatial derivatives in the acoustic wave system in equations 5 with SBP finite-difference operators, we arrive at the following semi-discretized system on a background block:

$$
\left\{\begin{aligned}
\mathcal{A}^{W_{x}} \boldsymbol{\rho}^{W_{x}} \frac{d W_{x}}{d t} & =\mathcal{A}^{W_{x}} \mathcal{D}_{x}^{P} P \\
\mathcal{A}^{W_{y}} \boldsymbol{\rho}^{W_{y}} \frac{d W_{y}}{d t} & =\mathcal{A}^{W_{y}} \mathcal{D}_{y}^{P} P \\
\mathcal{A}^{P} \mathcal{C}^{P} \frac{d P}{d t} & =\mathcal{A}^{P} \mathcal{D}_{x}^{W_{x}} W_{x}+\mathcal{A}^{P} \mathcal{D}_{y}^{W_{y}} W_{y} .
\end{aligned}\right.
$$

In equation 14. $W_{x}, W_{y}$, and $P$ are discrete solution vectors corresponding to solution variables $w_{x}, w_{y}$, and $p$, respectively; $\boldsymbol{\rho}^{W_{x}}, \boldsymbol{\rho}^{W_{y}}$, and $\mathcal{C}^{P}$ are diagonal matrices containing the discrete physical parameters on the respective subgrids, with $\boldsymbol{\rho}^{W_{x}}$ and $\boldsymbol{\rho}^{W_{y}}$ corresponding to density $\rho$ and $\mathcal{C}^{P}$ corresponding to compressibility $\frac{1}{\rho c^{2}} ; \mathcal{D}_{x}^{P}, \mathcal{D}_{y}^{P}, \mathcal{D}_{x}^{W_{x}}$, and $\mathcal{D}_{y}^{W_{y}}$ are the 2D SBP finite-difference operators whose superscripts indicate the solution variables that they act on and subscripts indicate the directions of differentiation; finally, $\mathcal{A}^{W_{x}}, \mathcal{A}^{W_{y}}$, and $\mathcal{A}^{P}$ are the 2D norm matrices, which are redundant in equation 14 , but will play an important role in the upcoming discrete energy analysis and in derivation of the proper interface treatment. The 2D SBP operators appearing in equation 14 are constructed from the previously introduced 1D SBP operators via tensor product. Details of their construction, as well as the relationships that they satisfy, are included in Appendix C. 
The discrete energy associated with the semi-discretized system 14 is defined as

$$
\mathscr{E}^{A}=\frac{1}{2} W_{i}^{T}\left(\mathcal{A}^{W_{i}} \boldsymbol{\rho}^{W_{i}}\right) W_{i}+\frac{1}{2} P^{T}\left(\mathcal{A}^{P} \mathcal{C}^{P}\right) P
$$

where the Einstein summation convention applies only to the subscript index $i$. The above discrete energy $\mathscr{E}^{A}$ emulates the continuous energy $e^{A}$ defined in equation 7 , with the $2 \mathrm{D}$ norm matrices playing the role of the integrals in equation 7 .

Differentiating equation 15 with respect to time and substituting in equations $14 \mathrm{a}-14 \mathrm{c}$, we arrive at

$$
\frac{d \mathscr{E} A}{d t}=P^{T}\left[\mathcal{A}^{P} \mathcal{D}_{x}^{W_{x}}+\left(\mathcal{A}^{W_{x}} \mathcal{D}_{x}^{P}\right)^{T}\right] W_{x}+P^{T}\left[\mathcal{A}^{P} \mathcal{D}_{y}^{W_{y}}+\left(\mathcal{A}^{W_{y}} \mathcal{D}_{y}^{P}\right)^{T}\right] W_{y}
$$

Further substituting in the relations from equation $\mathrm{C}-2$ and applying the mixed-product property $\mathbb{I}$ of the tensor product operator, we arrive at

$$
\begin{aligned}
\frac{d \mathscr{E}^{A}}{d t}= & \frac{\left[P^{T}\left(\mathcal{E}_{x}^{R} \otimes \mathcal{I}_{y}^{N}\right)\right] \mathcal{A}_{y}^{N}\left[\left(\mathcal{P}_{x}^{R} \otimes \mathcal{I}_{y}^{N}\right)^{T} W_{x}\right]}{-\left[P^{T}\left(\mathcal{E}_{x}^{L} \otimes \mathcal{I}_{y}^{N}\right)\right] \mathcal{A}_{y}^{N}\left[\left(\mathcal{P}_{x}^{L} \otimes \mathcal{I}_{y}^{N}\right)^{T} W_{x}\right]} \\
& +\left[P^{T}\left(\mathcal{I}_{x}^{N} \otimes \mathcal{E}_{y}^{R}\right)\right] \mathcal{A}_{x}^{N}\left[\left(\mathcal{I}_{x}^{N} \otimes \mathcal{P}_{y}^{R}\right)^{T} W_{y}\right]-\left[P^{T}\left(\mathcal{I}_{x}^{N} \otimes \mathcal{E}_{y}^{L}\right)\right] \mathcal{A}_{x}^{N}\left[\left(\mathcal{I}_{x}^{N} \otimes \mathcal{P}_{y}^{L}\right)^{T} W_{y}\right]
\end{aligned}
$$

Recalling the effects of the selection and projection operators, which were introduced in equation 13 and symbolized by $\mathcal{E}$ and $\mathcal{P}$ therein, we recognize that the quantities inside the square brackets in equation 17 , for example,

$$
\left[P^{T}\left(\mathcal{E}_{x}^{R} \otimes \mathcal{I}_{y}^{N}\right)\right] \text { and }\left[\left(\mathcal{I}_{x}^{N} \otimes \mathcal{P}_{y}^{L}\right)^{T} W_{y}\right]
$$

are approximations of the corresponding solution variables on the edges of the block region under consideration. The specific edges are revealed by the superscripts and subscripts of the selection or projection operators involved. For example, the two terms in equation 18 approximate $p$ on the right edge and $w_{y}$ on the bottom edge, respectively.

With the above observation, we have that $\frac{d \mathscr{E} A}{d t}$ in equation 17 emulates its continuous counterpart $\frac{d e^{A}}{d t}$ in equation 8 , with the $1 \mathrm{D}$ norm matrices $\mathcal{A}_{x}^{N}$ and $\mathcal{A}_{y}^{N}$ playing the role of the line integral in equation 8. More specifically, the expression of $\frac{d \mathscr{E} A}{d t}$ reduces to the edges of the block region only, with the terms underlined with solid, wavy, dotted, and dashed lines in equation 17 corresponding to the right, left, top, and bottom edges, respectively.

\section{Elastic target region}

Similar to the acoustic case discussed above, by replacing the spatial derivatives in elastic wave equations 9 with SBP finite-difference operators, we arrive at the following semi-discretized system on the target region:

$\mathbb{I}$ For matrices of compatible sizes, the mixed-product property states $(A \otimes B)(C \otimes D)=(A C) \otimes(B D)$. 


$$
\left\{\begin{aligned}
\mathcal{A}^{V_{x}} \boldsymbol{\rho}^{V_{x}} \frac{d V_{x}}{d t} & =\mathcal{A}^{V_{x}} \mathcal{D}_{x}^{\Sigma_{x x}} \Sigma_{x x}+\mathcal{A}^{V_{x}} \mathcal{D}_{y}^{\Sigma_{x y}} \Sigma_{x y} \\
\mathcal{A}^{V_{y}} \boldsymbol{\rho}^{V_{y}} \frac{d V_{y}}{d t} & =\mathcal{A}^{V_{y}} \mathcal{D}_{x}^{\Sigma_{x y}} \Sigma_{x y}+\mathcal{A}^{V_{y}} \mathcal{D}_{y}^{\Sigma_{y y}} \Sigma_{y y} \\
\mathcal{A}^{\Sigma_{x x}} \mathcal{S}_{x x k l}^{\Sigma_{k l}} \frac{d \Sigma_{k l}}{d t} & =\mathcal{A}^{\Sigma_{x x}} \mathcal{D}_{x}^{V_{x}} V_{x} \\
\mathcal{A}^{\Sigma_{x y}} \mathcal{S}_{x y k l}^{\Sigma_{k l}} \frac{d \Sigma_{k l}}{d t} & =\frac{1}{2} \mathcal{A}^{\Sigma_{x y}}\left(\mathcal{D}_{x}^{V_{y}} V_{y}+\mathcal{D}_{y}^{V_{x}} V_{x}\right) \\
\mathcal{A}^{\Sigma_{y y}} \mathcal{S}_{y y k l}^{\Sigma_{k l}} \frac{d \Sigma_{k l}}{d t} & =\mathcal{A}^{\Sigma_{y y}} \mathcal{D}_{y}^{V_{y}} V_{y},
\end{aligned}\right.
$$

where the Einstein summation convention applies only to the subscript indices $k$ and $l$ in equations 19c-19e. Treatment of the elastic-acoustic interfaces, i.e., boundaries of the target region, has been neglected in the above discretization and will be addressed in the next section. In equations $19, V_{x}$ and $V_{y}$ are discrete solution vectors corresponding to particle velocities $v_{x}$ and $v_{y}$ from equation 9 , respectively; $\Sigma_{k l}$ is the discrete solution vector corresponding to the stress component $\sigma_{k l}$ from equation 9 and finally, diagonal matrix $\mathcal{S}_{i j k l}^{\Sigma_{k l}}$ contains the discrete physical parameters corresponding to the compliance tensor component $s_{i j k l}$ from equation 9, with its superscript ${ }^{\Sigma_{k l}}$ indicating the subgrid that it is associated with. The 2D SBP operators appearing in equation 19 have similar meanings to those from equation 14 and are constructed in the same manner. Details of their construction, as well as the relationships that they satisfy, are included in Appendix C.

The discrete energy associated with the semi-discretized system 19 is defined as

$$
\mathscr{E}^{E}=\frac{1}{2} V_{i}^{T}\left(\mathcal{A}^{V_{i}} \boldsymbol{\rho}^{V_{i}}\right) V_{i}+\frac{1}{2} \Sigma_{i j}^{T}\left(\mathcal{A}^{\Sigma_{i j}} S_{i j k l}^{\Sigma_{k l}}\right) \Sigma_{k l}
$$

where the Einstein summation convention applies only to the subscript indices $i, j, k$, and $l$. The above discrete energy $\mathscr{E}^{E}$ emulates the continuous energy $e^{E}$ defined in equation 11 , with the $2 \mathrm{D}$ norm matrices playing the role of the integrals in equation 11 .

Differentiating equation 20 with respect to time and then substituting in equations $19 \mathrm{a}$ - $19 \mathrm{e}$ as well as the relations from equation $\mathrm{C}-4$, we eventually arrive at

$$
\begin{aligned}
& \frac{d \mathscr{E}^{E}}{d t}=\underline{\left[\Sigma_{x x}^{T}\left(\mathcal{E}_{x}^{R} \otimes \mathcal{I}_{y}^{N}\right)\right] \mathcal{A}_{y}^{N}\left[\left(\mathcal{P}_{x}^{R} \otimes \mathcal{I}_{y}^{N}\right)^{T} V_{x}\right]-\left[\Sigma_{x x}^{T}\left(\mathcal{E}_{x}^{L} \otimes \mathcal{I}_{y}^{N}\right)\right] \mathcal{A}_{y}^{N}\left[\left(\mathcal{P}_{x}^{L} \otimes \mathcal{I}_{y}^{N}\right)^{T} V_{x}\right]} \\
& +\underline{\left[V_{y}^{T}\left(\mathcal{E}_{x}^{R} \otimes \mathcal{I}_{y}^{M}\right)\right] \mathcal{A}_{y}^{M}\left[\left(\mathcal{P}_{x}^{R} \otimes \mathcal{I}_{y}^{M}\right)^{T} \Sigma_{x y}\right]}-\left[V_{y}^{T}\left(\mathcal{E}_{x}^{L} \otimes \mathcal{I}_{y}^{M}\right)\right] \mathcal{A}_{y}^{M}\left[\left(\mathcal{P}_{x}^{L} \otimes \mathcal{I}_{y}^{M}\right)^{T} \Sigma_{x y}\right] \\
& +\left[\Sigma_{y y}^{T}\left(\mathcal{I}_{x}^{N} \otimes \mathcal{E}_{y}^{R}\right)\right] \mathcal{A}_{x}^{N}\left[\left(\mathcal{I}_{x}^{N} \otimes \mathcal{P}_{y}^{R}\right)^{T} V_{y}\right]-\left[\Sigma_{y y}^{T}\left(\mathcal{I}_{x}^{N} \otimes \mathcal{E}_{y}^{L}\right)\right] \mathcal{A}_{x}^{N}\left[\left(\mathcal{I}_{x}^{N} \otimes \mathcal{P}_{y}^{L}\right)^{T} V_{y}\right]
\end{aligned}
$$

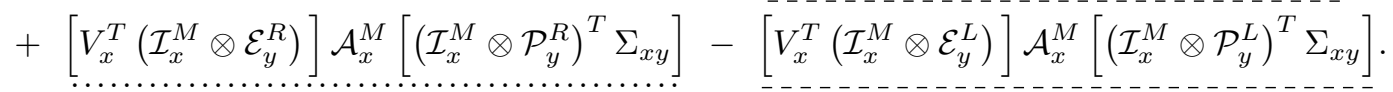

Similar to the acoustic case discussed before, the quantities inside the square brackets in equation 21 are approximations of the corresponding solution variables on the edges of the target region.

Consequently, we have that $\frac{d \mathscr{E}^{E}}{d t}$ in equation 21 emulates its continuous counterpart $\frac{d e^{E}}{d t}$ in equation 12, with the $1 \mathrm{D}$ norm matrices $\mathcal{A}_{x}^{N}, \mathcal{A}_{x}^{M}, \mathcal{A}_{y}^{N}$, and $\mathcal{A}_{y}^{M}$ playing the role of the line integral in equation 12 . More specifically, the expression of $\frac{d \mathscr{E}^{E}}{d t}$ reduces to the edges 
of the target region only, with the terms underlined with solid, wavy, dotted, and dashed lines corresponding to the right, left, top, and bottom edges, respectively.

\section{Interface treatment}

On elastic-acoustic interfaces, we seek to impose the following interface conditions, cf. (Stein and Wysession, 2009, p. 52),

$$
w_{i} n_{i}^{A}+v_{i} n_{i}^{E}=0
$$

and

$$
p n_{i}^{A}+\sigma_{i j} n_{j}^{E}=0
$$

where $n_{i}^{A}$ and $n_{i}^{E}$ (or $n_{j}^{E}$ ) represent components of the outward normal direction vectors at the interfaces from the acoustic and the elastic regions, respectively, which are the opposite of each other.

Recalling the results from equations 8 and 12 , and neglecting the exterior boundaries for now, we have that

$$
\frac{d e^{A}}{d t}+\frac{d e^{E}}{d t}=\int_{\partial \Omega_{T}} p w_{i} n_{i}^{A} d_{\partial \Omega_{T}}+\int_{\partial \Omega_{T}} v_{i} \sigma_{i j} n_{j}^{E} d_{\partial \Omega_{T}}=0
$$

after first applying the interface condition $22 \mathrm{~b}$, then using the fact that $n_{i}^{A}$ and $n_{i}^{E}$ are the opposite of each other, and, finally, applying the interface condition 22a, Equation 23 reveals that the total continuous energy, i.e., the sum of $e^{A}$ and $e^{E}$, is conserved across the elastic-acoustic interfaces. As demonstrated below, this property is preserved in the discretization with the proposed interface treatment.

It suffices to illustrate the interface treatment for the left edge of the target region as shown in Figure 1a, since the other edges can be addressed in a similar manner. This left edge is referred to as $T_{1} T_{4}$ using its two endpoints. For its treatment, we only need to consider the target region and the background block region left-adjacent to it, which share edge $T_{1} T_{4}$ as their interface.

To account for the interface conditions in equation 22 on edge $T_{1} T_{4}$, we append penalty terms, i.e., SATs, to the semi-discretized systems 14 and 19 , leading to the following modified semi-discretized systems 24 and 25, respectively,

(Acoustic)

$$
\left\{\begin{aligned}
\mathcal{A}^{W_{x}} \boldsymbol{\rho}^{W_{x}} \frac{d W_{x}}{d t} & =\mathcal{A}^{W_{x}} \mathcal{D}_{x}^{P} P \\
& +\eta_{A}^{W_{x}}\left[\mathcal{P}_{x}^{R} \otimes \mathcal{I}_{y}^{N}\right] \mathcal{A}_{y}^{N}\left\{\left[\left(\mathcal{E}_{x}^{R}\right)^{T} \otimes \mathcal{I}_{y}^{N}\right] P-\left[\left(\mathcal{E}_{x}^{L}\right)^{T} \otimes \mathcal{I}_{y}^{N}\right] \Sigma_{x x}\right\} \\
\mathcal{A}^{W_{y}} \boldsymbol{\rho}^{W_{y}} \frac{d W_{y}}{d t} & =\mathcal{A}^{W_{y}} \mathcal{D}_{y}^{P} P \\
\mathcal{A}^{P} \mathcal{C}^{P} \frac{d P}{d t} & =\mathcal{A}^{P} \mathcal{D}_{x}^{W_{x}} W_{x}+\mathcal{A}^{P} \mathcal{D}_{y}^{W_{y}} W_{y} \\
& +\eta_{A}^{P}\left[\mathcal{E}_{x}^{R} \otimes \mathcal{I}_{y}^{N}\right] \mathcal{A}_{y}^{N}\left\{\left[\left(\mathcal{P}_{x}^{R}\right)^{T} \otimes \mathcal{I}_{y}^{N}\right] W_{x}-\left[\left(\mathcal{P}_{x}^{L}\right)^{T} \otimes \mathcal{I}_{y}^{N}\right] V_{x}\right\}
\end{aligned}\right.
$$


(Elastic)

$$
\left\{\begin{aligned}
\mathcal{A}^{V_{x}} \boldsymbol{\rho}^{V_{x}} \frac{d V_{x}}{d t} & =\mathcal{A}^{V_{x}} \mathcal{D}_{x}^{\Sigma_{x x}} \Sigma_{x x}+\mathcal{A}^{V_{x}} \mathcal{D}_{y}^{\Sigma_{x y} \Sigma_{x y}} \\
& +\eta_{E}^{V_{x}}\left[\mathcal{P}_{x}^{L} \otimes \mathcal{I}_{y}^{N}\right] \mathcal{A}_{y}^{N}\left\{\left[\left(\mathcal{E}_{x}^{L}\right)^{T} \otimes \mathcal{I}_{y}^{N}\right] \Sigma_{x x}-\left[\left(\mathcal{E}_{x}^{R}\right)^{T} \otimes \mathcal{I}_{y}^{N}\right] P\right\} \\
\mathcal{A}^{V_{y}} \boldsymbol{\rho}^{V_{y}} \frac{d V_{y}}{d t} & =\mathcal{A}^{V_{y}} \mathcal{D}_{x}^{\Sigma_{x y}} \Sigma_{x y}+\mathcal{A}^{V_{y}} \mathcal{D}_{y}^{\Sigma_{y y} \Sigma_{y y}} \\
& +\eta_{E}^{V_{y}}\left[\mathcal{E}_{x}^{L} \otimes \mathcal{I}_{y}^{M}\right] \mathcal{A}_{y}^{M}\left\{\left[\left(\mathcal{P}_{x}^{L}\right)^{T} \otimes \mathcal{I}_{y}^{M}\right] \Sigma_{x y}-\mathbf{0}\right\} \\
\mathcal{A}^{\Sigma_{x x}} \mathcal{S}_{x x k l}^{\Sigma_{k l}} \frac{d \Sigma_{k l}}{d t} & =\mathcal{A}^{\Sigma_{x x}} \mathcal{D}_{x}^{V_{x}} V_{x} \\
& +\eta_{E}^{\Sigma_{x x}}\left[\mathcal{E}_{x}^{L} \otimes \mathcal{I}_{y}^{N}\right] \mathcal{A}_{y}^{N}\left\{\left[\left(\mathcal{P}_{x}^{L}\right)^{T} \otimes \mathcal{I}_{y}^{N}\right] V_{x}-\left[\left(\mathcal{P}_{x}^{R}\right)^{T} \otimes \mathcal{I}_{y}^{N}\right] W_{x}\right\} \\
\mathcal{A}^{\Sigma_{x y}} \mathcal{S}_{x y k l}^{\Sigma_{k l}} \frac{d \Sigma_{k l}}{d t} & =\frac{1}{2} \mathcal{A}^{\Sigma_{x y}}\left(\mathcal{D}_{x}^{V_{y}} V_{y}+\mathcal{D}_{y}^{V_{x}} V_{x}\right) \\
\mathcal{A}^{\Sigma_{y y}} \mathcal{S}_{y y k l}^{\Sigma_{k l}} \frac{d \Sigma_{k l}}{d t} & =\mathcal{A}^{\Sigma_{y y}} \mathcal{D}_{y}^{V_{y}} V_{y} .
\end{aligned}\right.
$$

We note here that the modified system of equations 24 applies only to the background block region left-adjacent to the target region. In these two modified semi-discretized systems, the penalty terms in equations $24 \mathrm{c}$ and $25 \mathrm{c}$ account for the interface condition stated in equation 22a, while the penalty terms in equations 24a and 25a, as well as equation 25b, account for the interface condition stated in equation $22 \mathrm{~b}$.

The scalars $\eta_{A}^{W_{x}}$ and $\eta_{A}^{P}$ from equation 24, as well as $\eta_{E}^{V_{x}}, \eta_{E}^{V_{y}}$ and $\eta_{E}^{\Sigma_{x x}}$ from equation 25, are penalty parameters. By setting them to be

$$
\eta_{A}^{W_{x}}=\eta_{A}^{P}=-1 / 2, \quad \eta_{E}^{V_{x}}=\eta_{E}^{\Sigma_{x x}}=1 / 2, \text { and } \eta_{E}^{V_{y}}=1,
$$

the energy-conserving property across edge $T_{1} T_{4}$ is preserved in the semi-discretization.

To verify this, we differentiate the sum of $\mathscr{E}^{A}$ and $\mathscr{E}^{E}$ with respect to time, similarly to what we did before in equations 17 and 21. However, this time, we only keep track of those terms that are associated with edge $T_{1} T_{4}$. After substituting in the equations from 24 and 25, we arrive at

$$
\begin{aligned}
& \left.\left(\frac{d \mathscr{E}^{A}}{d t}+\frac{d \mathscr{E}^{E}}{d t}\right)\right|_{T_{1} T_{4}} \\
& =\underline{\left[P^{T}\left(\mathcal{E}_{x}^{R} \otimes \mathcal{I}_{y}^{N}\right)\right] \mathcal{A}_{y}^{N}\left[\left(\mathcal{P}_{x}^{R} \otimes \mathcal{I}_{y}^{N}\right)^{T} W_{x}\right]} \\
& -\quad \underline{\left[\Sigma_{x x}^{T}\left(\mathcal{E}_{x}^{L} \otimes \mathcal{I}_{y}^{N}\right)\right] \mathcal{A}_{y}^{N}\left[\left(\mathcal{P}_{x}^{L} \otimes \mathcal{I}_{y}^{N}\right)^{T} V_{x}\right]}-\underline{\underline{\left[V_{y}^{T}\left(\mathcal{E}_{x}^{L} \otimes \mathcal{I}_{y}^{M}\right)\right] \mathcal{A}_{y}^{M}\left[\left(\mathcal{P}_{x}^{L} \otimes \mathcal{I}_{y}^{M}\right)^{T} \Sigma_{x y}\right]}} \\
& +\eta_{A}^{W_{x}} \underline{\left[W_{x}^{T}\left(\mathcal{P}_{x}^{R} \otimes \mathcal{I}_{y}^{N}\right)\right] \mathcal{A}_{y}^{N}\left[\left(\mathcal{E}_{x}^{R} \otimes \mathcal{I}_{y}^{N}\right)^{T} P\right]}-\eta_{A}^{W_{x}}\left[W_{x}^{T}\left(\mathcal{P}_{x}^{R} \otimes \mathcal{I}_{y}^{N}\right)\right] \mathcal{A}_{y}^{N}\left[\left(\mathcal{E}_{x}^{L} \otimes \mathcal{I}_{y}^{N}\right)^{T} \Sigma_{x x}\right] . \\
& +\eta_{A}^{P}\left[P^{T}\left(\mathcal{E}_{x}^{R} \otimes \mathcal{I}_{y}^{N}\right)\right] \mathcal{A}_{y}^{N}\left[\left(\mathcal{P}_{x}^{R} \otimes \mathcal{I}_{y}^{N}\right)^{T} W_{x}\right]-\eta_{A}^{P}\left[P^{T}\left(\mathcal{E}_{x}^{R} \otimes \mathcal{I}_{y}^{N}\right)\right] \mathcal{A}_{y}^{N}\left[\left(\mathcal{P}_{x}^{L} \otimes \mathcal{I}_{y}^{N}\right)^{T} V_{x}\right] \\
& +\eta_{E}^{V_{x}}\left[V_{x}^{T}\left(\mathcal{P}_{x}^{L} \otimes \mathcal{I}_{y}^{N}\right)\right] \mathcal{A}_{y}^{N}\left[\left(\mathcal{E}_{x}^{L} \otimes \mathcal{I}_{y}^{N}\right)^{T} \Sigma_{x x}\right]-\eta_{E}^{V_{x}}\left[V_{x}^{T}\left(\mathcal{P}_{x}^{L} \otimes \mathcal{I}_{y}^{N}\right)\right] \mathcal{A}_{y}^{N}\left[\left(\mathcal{E}_{x}^{R} \otimes \mathcal{I}_{y}^{N}\right)^{T} P\right] \\
& +\eta_{E}^{V_{y}} \underline{\underline{\left[V_{y}^{T}\left(\mathcal{E}_{x}^{L} \otimes \mathcal{I}_{y}^{M}\right)\right] \mathcal{A}_{y}^{M}\left[\left(\mathcal{P}_{x}^{L} \otimes \mathcal{I}_{y}^{M}\right)^{T} \Sigma_{x y}\right]}} \\
& +\eta_{E}^{\Sigma_{x x}}\left[\Sigma_{x x}^{T}\left(\mathcal{E}_{x}^{L} \otimes \mathcal{I}_{y}^{N}\right)\right] \mathcal{A}_{y}^{N}\left[\left(\mathcal{P}_{x}^{L} \otimes \mathcal{I}_{y}^{N}\right)^{T} V_{x}\right]-\eta_{E}^{\Sigma_{x x}}\left[\Sigma_{x x}^{T}\left(\mathcal{E}_{x}^{L} \otimes \mathcal{I}_{y}^{N}\right)\right] \mathcal{A}_{y}^{N}\left[\left(\mathcal{P}_{x}^{R} \otimes \mathcal{I}_{y}^{N}\right)^{T} W_{x}\right],
\end{aligned}
$$


where the term in line 27a stems from the acoustic background block region as in equation 17, the terms in line 27b stem from the elastic target region as in equation 21, the terms in lines $27 \mathrm{c}-27 \mathrm{~g}$ stem from the introduced penalty terms in equations 24a, 24c, 25a, 25b, and 25c respectively.

In equation 27, the terms underlined with the same line style are the same and therefore can be collected together. It can be easily verified that, with the choices of penalty parameters described in equation 26, the terms underlined with the same line styles cancel each other out. Consequently, we arrive at

$$
\left.\left(\frac{d \mathscr{E}^{A}}{d t}+\frac{d \mathscr{E}^{E}}{d t}\right)\right|_{T_{1} T_{4}}=0 .
$$

In other words, the semi-discretization presented in equations 24 and 25 preserves the energy-conserving property across edge $T_{1} T_{4}$.

Similar modifications to the semi-discretized systems presented in equations 14 and 19 can be made for the other three edges. To conserve space, these modifications are provided in the supplementary material. Observations similar to that in equation 28 can be made for the other three edges as well. After all these modifications, it can be shown that all the terms in equations 17 and 21 that are associated with the elastic-acoustic interfaces get cancelled out by the introduction of the properly designed penalty terms.

Furthermore, with proper exterior boundary conditions, e.g., the free-surface boundary condition $p=0$, the total continuous energy $e^{A}+e^{E}$ is conserved over the entire simulation domain, as evidenced from equations 8 and 23 . When combined with proper treatment of these exterior boundaries as well as the acoustic-acoustic artificial interfaces, cf. Gao et al. (2019), the overall discretization using the elastic-acoustic interface treatment described above can be shown to conserve the total discrete energy $\mathscr{E}^{A}+\mathscr{E}^{E}$ over the entire simulation domain. Such property will be demonstrated below.

As a side note, we point out here that when used to couple different parallel subdomains, the SBP-SAT technique leads to the desired features of low communication volume and simple communication pattern. Taking the pair of equations 24a and 25a for example, the quantity that needs to be communicated from the elastic region to the acoustic region is $\left[\left(\mathcal{E}_{x}^{L}\right)^{T} \otimes \mathcal{I}_{y}^{N}\right] \Sigma_{x x}$, while $\left[\left(\mathcal{E}_{x}^{R}\right)^{T} \otimes \mathcal{I}_{y}^{N}\right] P$ is the quantity that needs to be communicated reversely; both are restricted to the interface.

\section{NUMERICAL EXAMPLES}

In the following, we demonstrate the efficacy of the presented elastic-acoustic interface treatment with numerical examples. For all the tests presented below, the staggered leapfrog scheme is used for time integration. The initial status of the medium is assumed to be quiescent, which means that all solution components and their derivatives are zero at the beginning of the simulation. The wave propagation is driven by a point source term that mimics the explosive source commonly used in seismic exploration surveys. 


\section{Example 1. Validation against analytical solution}

In this example, we validate the presented interface treatment with the semi-analytical solution provided at http://www.spice-rtn.org/library/software/EX2DELEL.html by the SPICE project. A horizontal elastic-acoustic interface is considered, which separates the acoustic medium above it and the elastic medium below it. Both media are homogeneous. The acoustic medium is characterized by compressional wave-speed $c_{p}^{A}=3 \mathrm{~m} / \mathrm{s}$ and density $\rho^{A}=1 \mathrm{~kg} / \mathrm{m}^{3}$; the elastic medium is characterized by compressional wave-speed $c_{p}^{E}=9 \mathrm{~m} / \mathrm{s}$, shear wave-speed $c_{s}^{E}=5 \mathrm{~m} / \mathrm{s}$, and density $\rho^{E}=2 \mathrm{~kg} / \mathrm{m}^{3}$.

Both source and receiver are placed in the acoustic medium. Specifically, the source is at $0.15 \mathrm{~m}$ above the interface on the $P$ subgrid; the receiver is at $0.1575 \mathrm{~m}$ above the interface and $12 \mathrm{~m}$ to the right of the source on the $W_{y}$ subgrid. The source temporal profile is chosen as the Ricker wavelet with $5 \mathrm{~Hz}$ central frequency and $0.25 \mathrm{~s}$ time delay. The maximal frequency in the source content is counted as $12.5 \mathrm{~Hz}$, which, together with the minimal wave-speed, i.e., $c_{p}^{A}=3 \mathrm{~m} / \mathrm{s}$, leads to minimal wavelength at $0.24 \mathrm{~m}$.

In the numerical simulation, the spatial grid spacing is chosen as $\Delta x=0.015 \mathrm{~m}$, which amounts to 16 grid points per minimal wavelength. The time step length is chosen as $\Delta t=$ $8 \mathrm{e}-4 \mathrm{~s}$. Time history of $W_{y}$ at the receiver location from the numerical simulation is recorded for $6 \mathrm{~s}$ and compared with the semi-analytical solution in Figure 3 . We observe that the two solutions agree very well with each other.

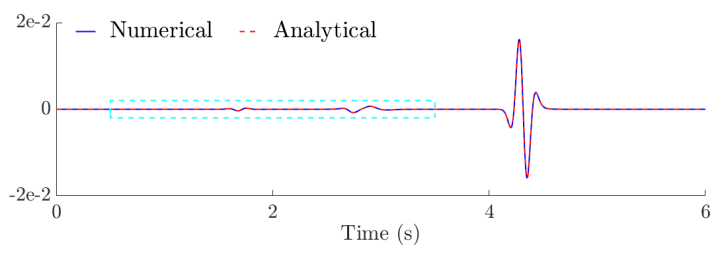

a)

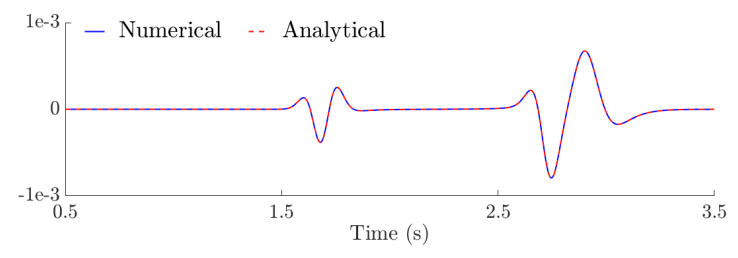

b)

Figure 3: Time history of $W_{y}$ at the receiver location from the numerical simulation compared with the semi-analytical solution. (a): the entire $6 \mathrm{~s}$ signal; (b): zoom-in of the segment between $0.5 \mathrm{~s}$ and $3.5 \mathrm{~s}$.

\section{Example 2. Homogeneous media}

In this example, we consider a simulation domain as illustrated in Figure 1a, where the elastic medium is surrounded by the acoustic medium. Both media are characterized by homogeneous parameters, which are the same as those specified in the previous example. The entire simulation domain is divided into nine subdomains, each discretized by a set of staggered grids, cf. Figure $1 \mathrm{~b}$. On each subdomain, there are $101 \times 101$ grid points on the $N$ subgrid, which is occupied by solution variable $p$ on an acoustic subdomain and by normal stresses $\sigma_{x x}$ and $\sigma_{y y}$ on the elastic subdomain. An interface shared by two subdomains is duplicated and included in the discretizations of both subdomains.

The free-surface boundary condition, i.e., $p=0$, is considered for all exterior boundaries, which is also imposed via the SBP-SAT technique. Interested readers may refer to Gao et al. (2019) for more detail. This choice enables us to demonstrate the energy-conserving 
property of the proposed interface treatment since there is no energy loss at the free-surface exterior boundaries.

A point source is placed at the center of the bottom left subdomain while a receiver is placed at the center of the top right subdomain, both on the $N$-subgrid. The source temporal profile is the same as in the previous example. Time history of $P$ at the receiver location is recorded during the simulation and used later for comparison. The spatial grid spacing is again chosen as $\Delta x=0.015 \mathrm{~m}$, which amounts to 16 grid points per minimal wavelength. The time step length is chosen as $\Delta t \approx 9.428 \mathrm{e}-4 \mathrm{~s}$, which corresponds to a Courant number 0.8. Duration of the total simulation is set to $6 \mathrm{~s}$.

The recorded time history of $P$ at the receiver location from the coupled simulation is displayed in Figure 4 and labeled as 'Coupled'. For comparison, also shown in Figure 4 are the full elastic simulation results, which use the elastic wave system for the entire simulation domain and simply treat the acoustic medium in the background region as a special case of the elastic medium whose shear wave-speed value is zero.

Discretization of the elastic wave system posed in the first-order form A-2 can be used to stably simulate wave propagation on acoustic medium. In fact, one can easily verify that, by setting the shear wave-speed $c_{s}$ to zero, equation A-2 reduces to equation A-1. To start, $\sigma_{x y}$ remains zero since the shear modulus $\mu=0$; both $\sigma_{x x}$ and $\sigma_{y y}$ replicate $p$ since both $\lambda$ and $\lambda+2 \mu$ reduce to the bulk modulus $\rho c^{2}$ in equation A-1; finally, with $\sigma_{x x}$ and $\sigma_{y y}$ replicating $p$ and $\sigma_{x y}$ being zero, $v_{x}$ and $v_{y}$ in equation A-2 reduce to $w_{x}$ and $w_{y}$ in equation A-1, respectively.

However, this implicit treatment of the elastic-acoustic interfaces will negatively affect the simulation accuracy since there is an infinite jump in the shear wave-speed value. Consequently, much finer grid spacing is needed to resolve the waves near the interfaces. In Figure 4, the full elastic simulation results using grid spacings that are 1/16 and 1/32 of that used in the coupled simulation are presented as reference solutions, which are labeled as 'Elastic $(16 \times)$ ' and 'Elastic $(32 \times)$ ', respectively. The close proximity between these two reference solutions indicates that the waves have been well resolved in these reference simulations, even near the interfaces.

From Figure 4, we observe that the coupled simulation result matches very well with the reference solutions as they overlay one another. In addition, evolution of the total discrete energy associated with the coupled simulation, i.e., the sum of $\mathscr{E}^{A}$ from equation 15 over the acoustic region and $\mathscr{E}^{E}$ from equation 20 over the elastic region, is plotted in Figure 5 , where we observe that the total discrete energy remains constant after the source term tapers off, which corroborates the energy-conserving property of the proposed interface treatment.

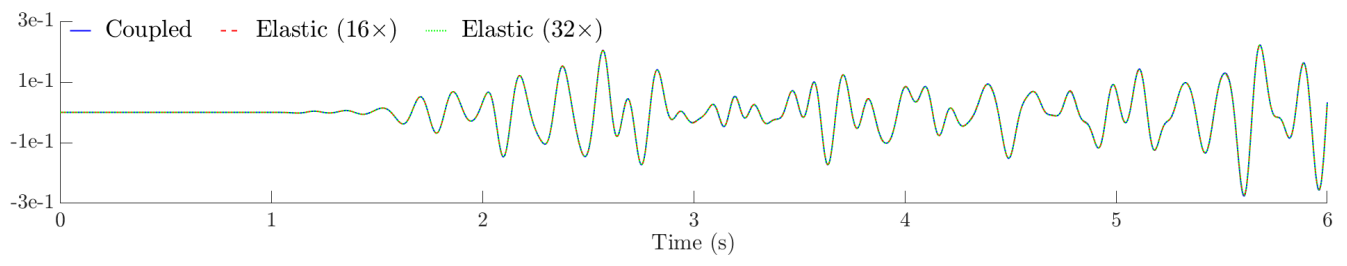

Figure 4: Time history of $P$ at the receiver location from the coupled simulation compared with full elastic simulation results. 


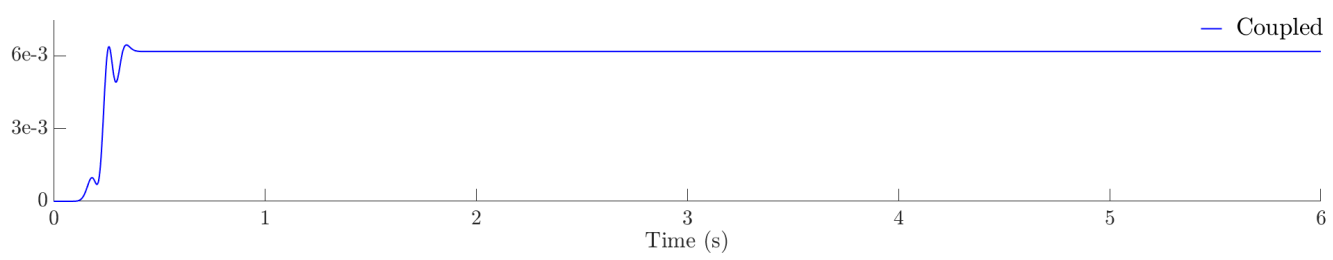

Figure 5: Evolution of the total discrete energy associated with the coupled simulation.

Since the coupled simulation and the elastic $(1 \times)$ simulation use the same spatial grid spacing $\Delta x$ and temporal step length $\Delta t$, the ratio of the computational costs associated with these two simulations can be estimated by the numbers of spatial derivative approximations at each grid point summed over the entire simulation domain. This number is 4 in the acoustic region and 8 in the elastic region, which leads to an estimated ratio of $\sim 55.6 \%$ for this example. In a serial setting, the elapsed times measured for the time loops in these two simulations are $\sim 24.85 \mathrm{~s}$ and $\sim 41.98 \mathrm{~s}$, respectively, with a ratio of $\sim 59.2 \%$. ${ }^{* *}$

\section{Example 3. Heterogeneous media}

In this example, we validate the proposed interface treatment with heterogeneous media. Specifically, we consider a vertical section of the 3D SEG/EAGE salt model (Aminzadeh et al., 1994). The compressional wave-speed is displayed in Figure 6, where the high velocity object in the center represents a salt body. This $2 \mathrm{D}$ domain is then divided into nine subdomains, with the subdomain in the center that encapsulates the salt body being treated as the elastic region and the remaining subdomains being treated as the acoustic region.

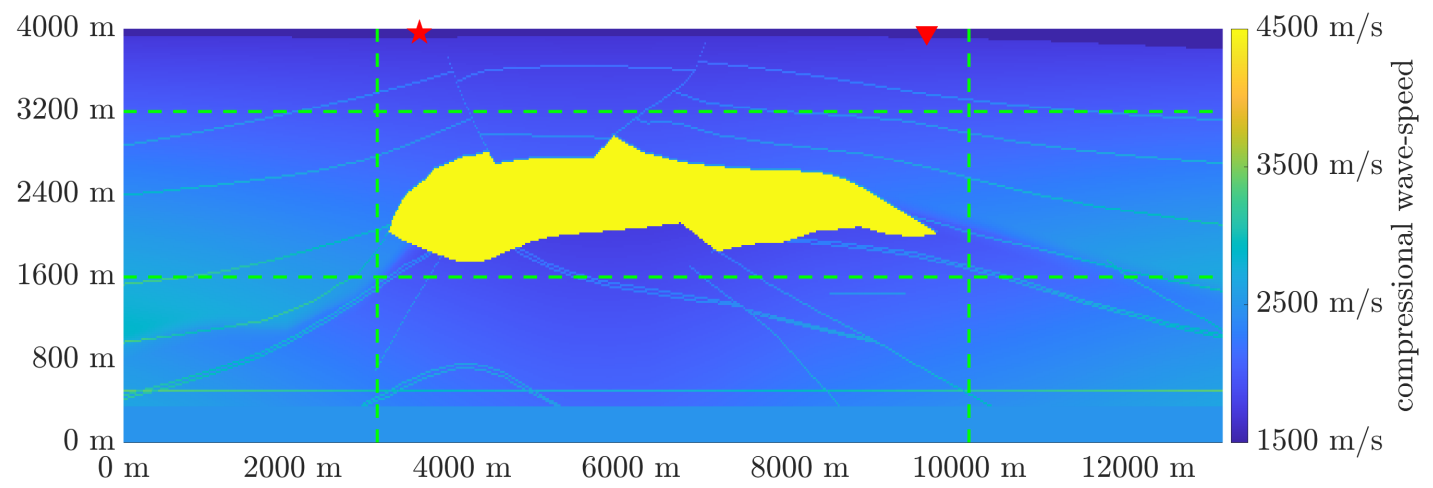

Figure 6: Illustration of the heterogeneous media (compressional wave-speed) used in Example 3. The source and receiver locations are indicated by the star and the triangle, respectively, with a distance of $6000 \mathrm{~m}$ in between. The green dashed lines mark the interfaces that separate different subdomains.

Since the original SEG/EAGE salt model contains only the compressional wave-speed information, we supplement the density and shear wave-speed information as follows. Start-

\footnotetext{
** Each simulation is run for 7 times. Omitting the maximum and minimum, average of the remaining 5 recorded elapsed times is reported here as the measured time. The executables are generated with the GCC compiler with -O3 optimization flag and re-compiled after each run. The coupled simulation is slightly slower than the estimate predicts. This is largely due to a suboptimal implementation of the interface treatment in our prototype code, which can be improved upon in future more performance-oriented implementations.
} 
ing at the bottom of the water layer, the density follows a linear function that increases with depth from $1000 \mathrm{~kg} / \mathrm{m}^{3}$ to $2500 \mathrm{~kg} / \mathrm{m}^{3}$. For the subdomain in the center, the shear wavespeed value is assigned based on the compressional wave-speed value using the following range-wise formula

$$
c_{s}= \begin{cases}1000 & \text { if } \quad c_{p} \leq 2200 \\ c_{p} / 2.15 & \text { if } 2200<c_{p} \leq 3500 \\ c_{p} / 1.75 & \text { if } 3500<c_{p}\end{cases}
$$

where $c_{p}$ and $c_{s}$ stand for compressional and shear wave-speed values, respectively, both in unit $\mathrm{m} / \mathrm{s}$. The remaining subdomains are not assigned with shear wave-speed values since they are treated as the acoustic region. Over the entire simulation domain, the minimal and maximal wave-speed values are $1000 \mathrm{~m} / \mathrm{s}$ and $4482 \mathrm{~m} / \mathrm{s}$, respectively.

With the media parameters described above, we are now ready to set up the numerical experiments. Specifically, we use the Ricker wavelet with $16 \mathrm{~Hz}$ central frequency and $0.125 \mathrm{~s}$ time delay as the source temporal profile. The maximal frequency in the source content is counted as $40 \mathrm{~Hz}$, which, together with the minimal wave-speed value $1000 \mathrm{~m} / \mathrm{s}$, leads to a minimal wavelength at $25 \mathrm{~m}$.

We assign 10 grid points per minimal wavelength for the coupled simulation, which then leads to grid spacing $\Delta x=2.5 \mathrm{~m}$ in the discretization grids. Since the discretization grid spacing $(2.5 \mathrm{~m})$ is smaller than the parametric grid spacing $(20 \mathrm{~m})$, we interpolate the medium parameters bilinearly from the parametric grid to the discretization grids. The time step length is chosen as $\Delta t \approx 3.155 \mathrm{e}-4 \mathrm{~s}$, which corresponds to a Courant number 0.8. Duration of the simulation is set to $6 \mathrm{~s}$.

The free-surface boundary condition, i.e., $p=0$, is again considered for all exterior boundaries so that we can verify the energy-conserving property of the proposed interface treatment. Finally, the source and receiver locations are depicted in Figure 6, with a distance of $6000 \mathrm{~m}$ in between.

As in the previous example, the recorded time history of $P$ at the receiver location from the coupled simulation is displayed in Figure 7 and compared with the full elastic simulation results. Zoom-in of the segment encircled by the dashed lines is displayed in Figure 8, which contains waves that arrive earlier than the main wave train in Figure 7 .

As evidenced by the snapshot shown in Figure 9, these early arrival waves travel through the salt body, which is the fast pathway due to the high wave-speeds associated with the salt body. Despite their relatively small amplitude, these early arrival waves are essential for salt body characterization and sub-salt imaging.

The well matched signals in Figures 7 and 8 validate the accuracy of the proposed interface treatment. ${ }^{\dagger \dagger}$ Moreover, evolution of the total discrete energy associated with the coupled simulation is displayed in Figure 10, which remains constant after the source term tapers off, and hence corroborates the energy-conserving property of the proposed interface treatment.

\footnotetext{
${ }^{\dagger \dagger}$ Appendix $\mathrm{D}$ contains two figures that are the equivalents of Figures 7 and 8 , respectively, with the receiver location placed just outside the top-right corner of the elastic region.
} 


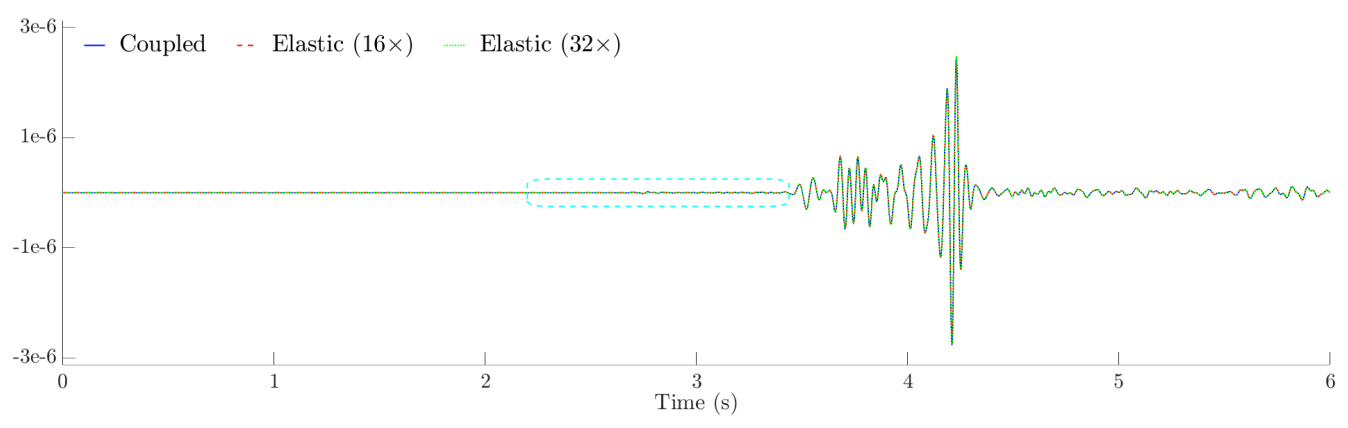

Figure 7: Time history of $P$ recorded at the receiver location from coupled simulation compared with full elastic simulation results.

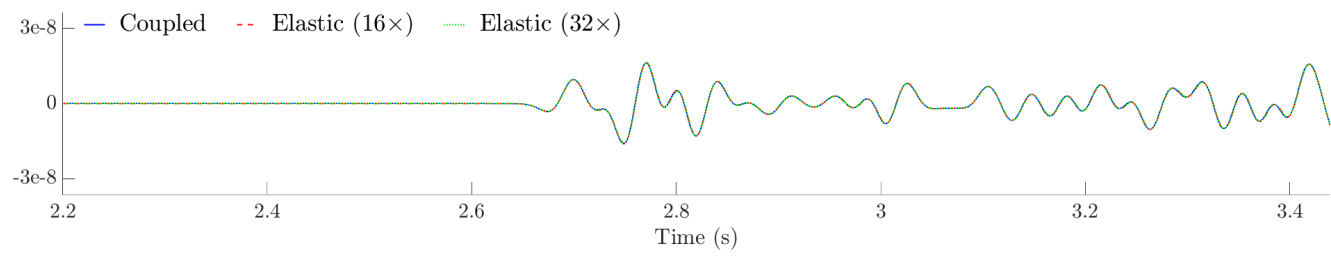

Figure 8: Zoom-in display of the segment encircled by the dashed lines in Figure 7 .

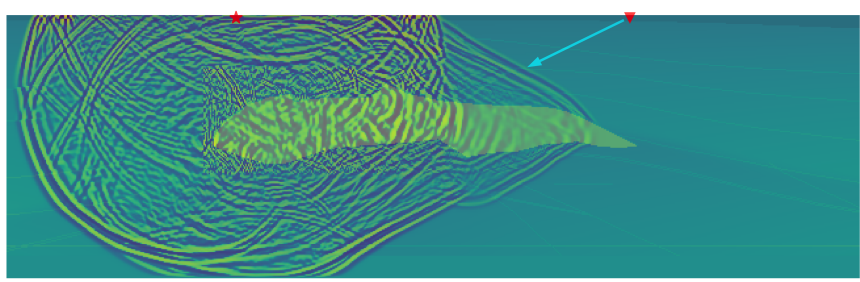

Figure 9: Snapshot of the wave-field (specifically, its vertical velocity component) at around $1.89 \mathrm{~s}$. It can be observed that the early arrival waves, pointed at by the arrow, 'radiate' from the salt body.

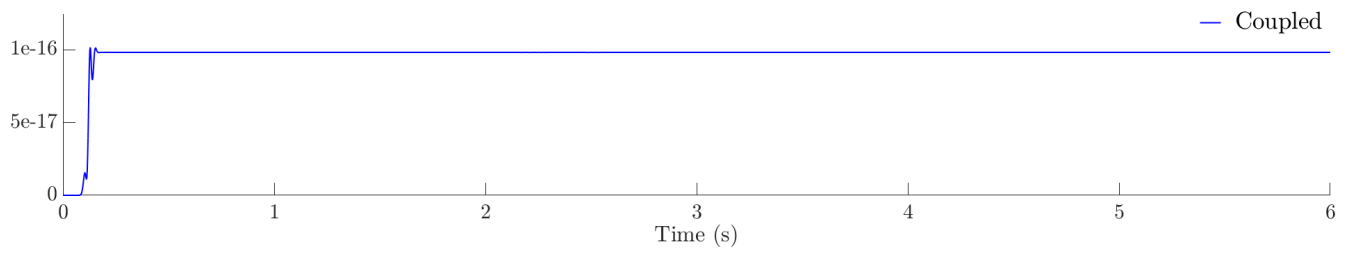

Figure 10: Evolution of the total discrete energy associated with the coupled simulation.

As comparison, the seismogram simulated with the full acoustic model is displayed in Figure 11, with the zoom-in of the early arrival waves displayed in Figure 12, Significant discrepancy can be observed in the early arrival waves, since the acoustic model is not able to properly capture the elastic properties of the salt body. 


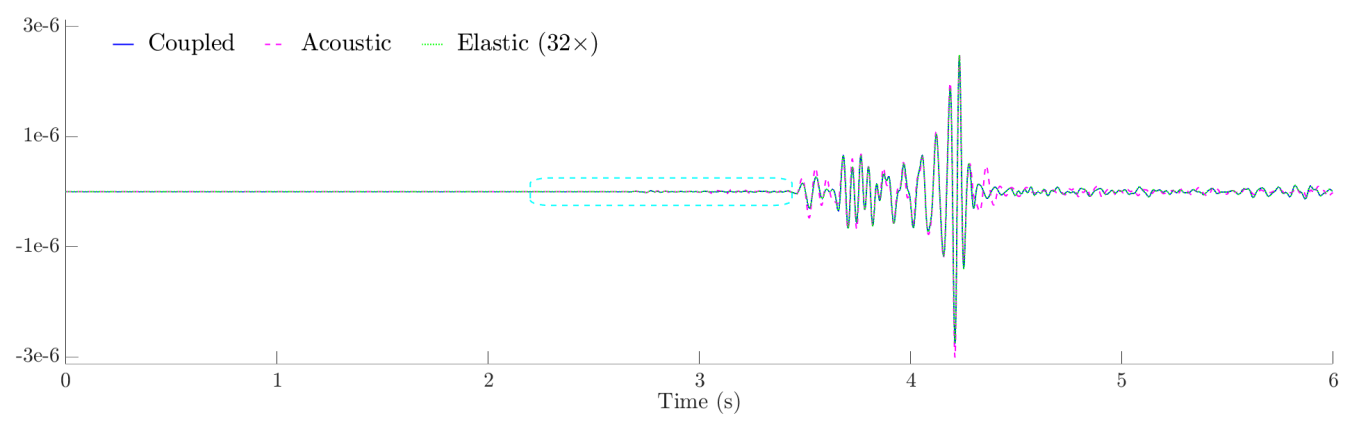

Figure 11: Time history of $P$ recorded at the receiver location from coupled, full acoustic and full elastic simulation results.

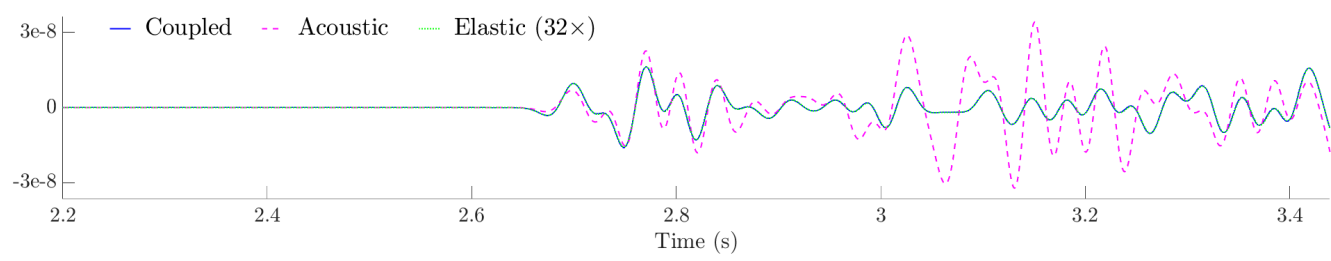

Figure 12: Zoom-in display of the segment encircled by the dashed lines in Figure 11

\section{DISCUSSION}

The above numerical examples validate the efficacy of the presented elastic-acoustic interface treatment and exhibit the potential of the coupled elastic-acoustic wave simulations for seismic imaging applications. However, this study is only the first step toward a concrete strategy to properly exploit the benefits of such coupled simulations in seismic imaging. A potential future extension is to use different discretization grid spacings for the elastic and acoustic regions, which will lead to nonconforming grid interfaces. Paired interpolation operators that satisfy special relations, cf., for example, equation 15 of Mattsson and Carpenter (2010) or equation 58 of Gao et al. (2019), are required to properly address nonconforming grid interfaces. Assisted by modern symbolic computing software, such interpolation operators can be derived without much difficulty. Extension to coupling nonconforming elastic-acoustic interfaces can then be achieved through straightforward modifications to the penalty terms presented above using these interpolation operators, similar to what has been presented in Gao et al. (2019) for the acoustic case. Additionally, similar discretization technique also based on staggered grid SBP finite-difference operators has recently been extended to curvilinear grids for the acoustic wave equation (O'Reilly and Petersson, 2020). This indicates that similar extension may also be attainable for the coupled acoustic elastic wave system, which can be applied to address the irregular geometry at the ocean bottom.

\section{CONCLUSION}

The problem of explicitly coupling the simulations of acoustic and isotropic elastic wave systems is considered. Such coupled simulation has applications in seismic exploration studies involving salt bodies, where the region surrounding the salt body can be modeled with the elastic wave system to account for the converted waves, while the background 
region can still be modeled with the computationally more affordable acoustic wave system. Energy-conserving numerical discretization based on the techniques of summation-by-parts finite-difference operators and simultaneous approximation terms has been derived for the coupled wave system. Specifically, the elastic-acoustic interface conditions are addressed by carefully designed simultaneous approximation terms that enforce these conditions weakly. Satisfactory simulation results have been demonstrated on both piece-wise homogeneous and generally heterogeneous media.

\section{ACKNOWLEDGMENTS}

The authors thank the editors and referees for carefully reviewing this article and providing valuable suggestions. Additionally, the authors would like to thank Thierry-Laurent Tonellot and Vincent Etienne (Saudi Aramco) for helpful discussions. For computer time, this research used the resources of the KAUST Supercomputing Laboratory. This research was funded by KAUST under the Extreme Computing Research Center, KAUST grant OSR-2019-CCF-3666.4, Saudi Aramco grant RGC/3/3226-01-01, and UT Austin.

\section{APPENDIX A}

\section{PRACTICAL IMPLEMENTATION}

As mentioned in the section Problem Description, the forms of the continuous acoustic and elastic wave systems presented in equations 5 and 9 are more convenient for energy analysis and formula derivation, while their equivalent forms shown in equations A-1 and A-2, respectively, are more suited for the actual implementation.

(Acoustic)

$$
\left\{\begin{aligned}
\frac{\partial w_{x}}{\partial t} & =\frac{1}{\rho} \frac{\partial p}{\partial x} \\
\frac{\partial w_{y}}{\partial t} & =\frac{1}{\rho} \frac{\partial p}{\partial y} \\
\frac{\partial p}{\partial t} & =\rho c^{2}\left(\frac{\partial w_{x}}{\partial x}+\frac{\partial w_{y}}{\partial y}\right)
\end{aligned}\right.
$$

(Elastic)

$$
\left\{\begin{aligned}
\frac{\partial v_{x}}{\partial t} & =\frac{1}{\rho}\left(\frac{\partial \sigma_{x x}}{\partial x}+\frac{\partial \sigma_{x y}}{\partial y}\right) \\
\frac{\partial v_{y}}{\partial t} & =\frac{1}{\rho}\left(\frac{\partial \sigma_{x y}}{\partial x}+\frac{\partial \sigma_{y y}}{\partial y}\right) \\
\frac{\partial \sigma_{x x}}{\partial t} & =(\lambda+2 \mu) \frac{\partial v_{x}}{\partial x}+\lambda \frac{\partial v_{y}}{\partial y} \\
\frac{\partial \sigma_{x y}}{\partial t} & =\mu \frac{\partial v_{y}}{\partial x}+\mu \frac{\partial v_{x}}{\partial y} \\
\frac{\partial \sigma_{y y}}{\partial t} & =\lambda \frac{\partial v_{x}}{\partial x}+(\lambda+2 \mu) \frac{\partial v_{y}}{\partial y}
\end{aligned}\right.
$$


After obtaining the modified semi-discretized systems presented in equations 24 and 25. where the interface conditions have been properly accounted for, one can easily convert these systems to forms that conform with equations A-1 and A-2 by viewing the appended penalty terms as modifications to the spatial derivative approximations.

For example, equation 24a can be re-written as

$$
\mathcal{A}^{W_{x}} \boldsymbol{\rho}^{W_{x}} \frac{d W_{x}}{d t}=\mathcal{A}^{W_{x}} \widetilde{\mathcal{D}_{x}^{P} P}
$$

where the modified derivative approximation $\widetilde{\mathcal{D}_{x}^{P P}}$ is defined as

$$
\widetilde{\mathcal{D}_{x}^{P} P}=\mathcal{D}_{x}^{P} P+\eta_{A}^{W_{x}}\left(\mathcal{A}^{W_{x}}\right)^{-1}\left[\mathcal{P}_{x}^{R} \otimes \mathcal{I}_{y}^{N}\right] \mathcal{A}_{y}^{N}\left\{\left[\left(\mathcal{E}_{x}^{R}\right)^{T} \otimes \mathcal{I}_{y}^{N}\right] P-\left[\left(\mathcal{E}_{x}^{L}\right)^{T} \otimes \mathcal{I}_{y}^{N}\right] \Sigma_{x x}\right\} .
$$

The norm matrix $\mathcal{A}^{W_{x}}$ can now be dropped from both sides of equation A-3. Going through the same procedure for the remaining equations in system 24, we end up with a semidiscretized system that has a term-by-term correspondence with system 5 , which can then be converted to the form that conforms with system A-1 by moving the coefficient matrices (e.g., $\boldsymbol{\rho}^{W_{x}}$ in equation $\mathrm{A}-3$ to the right hand side of the equations.

Similarly, equations 25 can be converted to the form that conforms with equations A-2 by following the same procedure. An extra complication, which stems from the tangled stress components in equations $9 \mathrm{c}-9 \mathrm{e}$, is that after moving the coefficient matrices to the right hand side of the equations, the modified derivative approximation $\widetilde{\mathcal{D}_{x}^{V_{x}} V_{x}}$ will appear in the updates of both $\Sigma_{x x}$ and $\Sigma_{y y}$, as illustrated below:

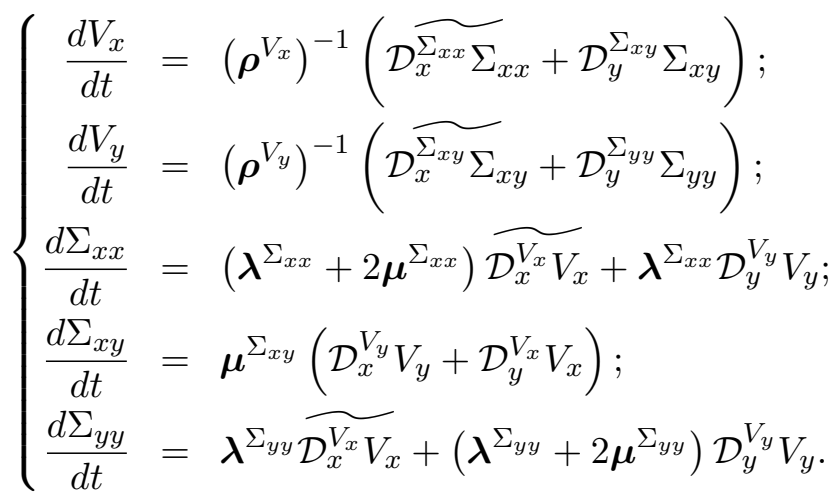

\section{APPENDIX B}

\section{THE 1D SBP OPERATORS}

The following 1D SBP operators in equations B-1a - B-1d serve as the building blocks for the finite-difference discretization presented in the section Spatial Discretization. They have already appeared in Gao et al. (2019) and are included here to make this work selfcontained. Interested readers may refer to Gao et al. (2019) for more detail. 


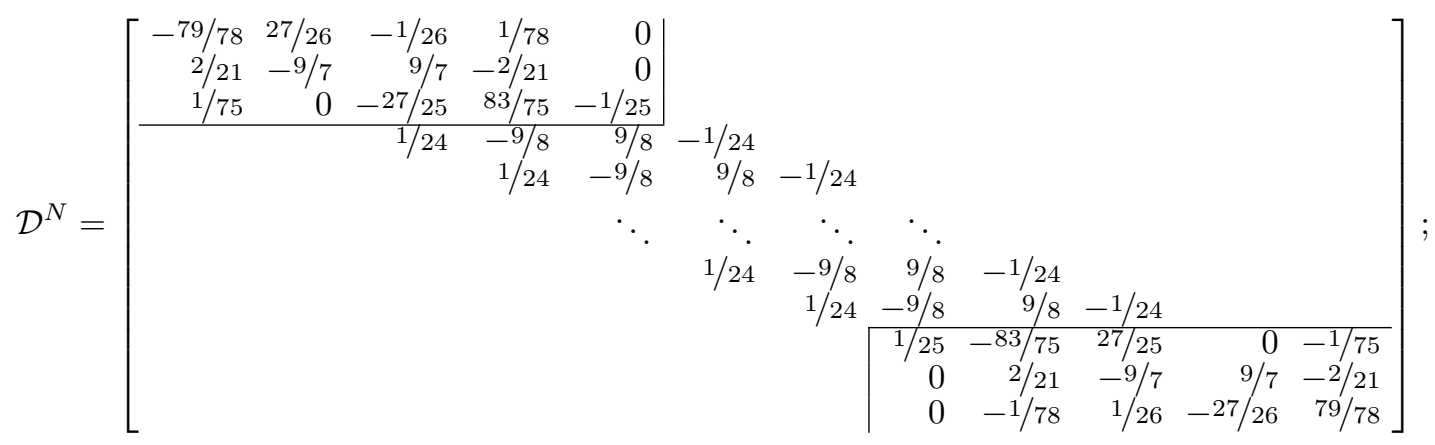

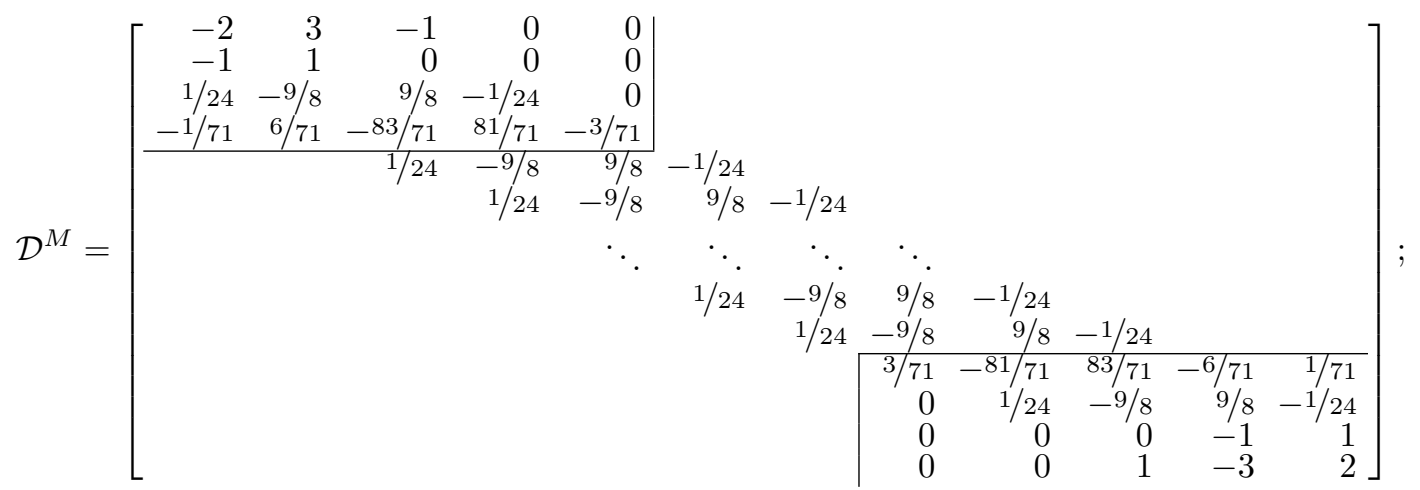

(B-1a)

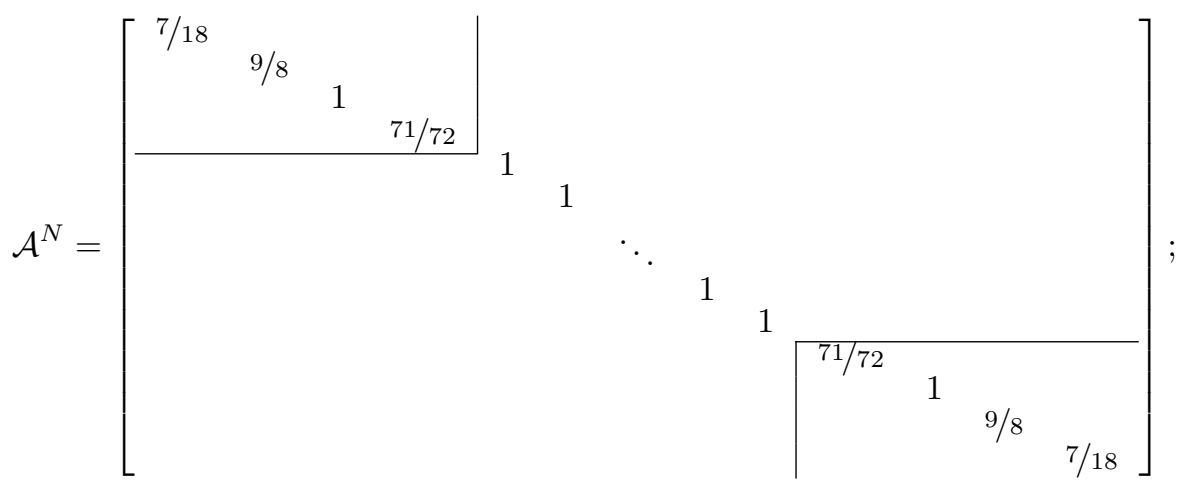

(B-1b)

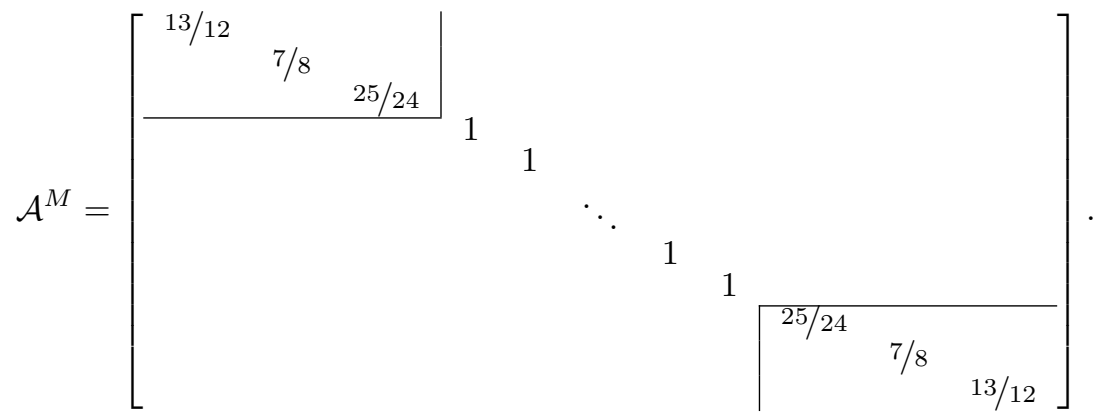

(B-1c)

We note here that the matrices presented in equation B-1 correspond to the case of unit grid spacing, i.e., $\Delta x=1$. When applied to general cases, $\mathcal{D}^{N}$ and $\mathcal{D}^{M}$ need to be scaled by $\frac{1}{\Delta x}$ while $\mathcal{A}^{N}$ and $\mathcal{A}^{M}$ need to be scaled by $\Delta x$.

With the above matrices, the expression $\mathcal{A}^{N} \mathcal{D}^{M}+\left(\mathcal{A}^{M} \mathcal{D}^{N}\right)^{T}$ from equation 13 takes 
the following form:

$$
\left[\begin{array}{rrrrrr}
-15 / 8 & 5 / 4 & -3 / 8 & & & \\
& & & & & \\
& & & & & \\
& & & 3 / 8 & -5 / 4 & 15 / 8
\end{array}\right],
$$

which can be recast as $\mathcal{E}^{R}\left(\mathcal{P}^{R}\right)^{T}-\mathcal{E}^{L}\left(\mathcal{P}^{L}\right)^{T}$ with $\mathcal{E}^{L}, \mathcal{E}^{R}, \mathcal{P}^{L}$ and $\mathcal{P}^{R}$ given by

$$
\mathcal{E}^{L}=\left[\begin{array}{c}
1 \\
0 \\
\vdots \\
0
\end{array}\right] ; \quad \mathcal{E}^{R}=\left[\begin{array}{c}
0 \\
\vdots \\
0 \\
1
\end{array}\right] ; \quad \mathcal{P}^{L}=\left[\begin{array}{c}
15 / 8 \\
-5 / 4 \\
3 / 8 \\
0 \\
\vdots \\
0
\end{array}\right] ; \quad \mathcal{P}^{R}=\left[\begin{array}{c}
0 \\
\vdots \\
0 \\
3 / 8 \\
-5 / 4 \\
15 / 8
\end{array}\right],
$$

respectively.

\section{APPENDIX C}

\section{THE 2D SBP OPERATORS}

The 2D SBP operators appearing in the semi-discretized acoustic wave system 14 are constructed from the 1D SBP operators via tensor product as follows

and

$$
\mathcal{A}^{W_{x}}=\mathcal{A}_{x}^{M} \otimes \mathcal{A}_{y}^{N}, \quad \mathcal{A}^{W_{y}}=\mathcal{A}_{x}^{N} \otimes \mathcal{A}_{y}^{M}, \mathcal{A}^{P}=\mathcal{A}_{x}^{N} \otimes \mathcal{A}_{y}^{N}
$$

$$
\mathcal{D}_{x}^{W_{x}}=\mathcal{D}_{x}^{M} \otimes \mathcal{I}_{y}^{N}, \mathcal{D}_{x}^{P}=\mathcal{D}_{x}^{N} \otimes \mathcal{I}_{y}^{N}, \mathcal{D}_{y}^{W_{y}}=\mathcal{I}_{x}^{N} \otimes \mathcal{D}_{y}^{M}, \mathcal{D}_{y}^{P}=\mathcal{I}_{x}^{N} \otimes \mathcal{D}_{y}^{N},
$$

where $\otimes$ stands for the tensor product operation while $\mathcal{I}$ symbolizes a 1D identity matrix. Constructed as such, these 2D SBP operators satisfy the following relations:

$$
\begin{aligned}
& \mathcal{A}^{P} \mathcal{D}_{x}^{W_{x}}+\left(\mathcal{A}^{W_{x}} \mathcal{D}_{x}^{P}\right)^{T}=\left[\mathcal{A}_{x}^{N} \mathcal{D}_{x}^{M}+\left(\mathcal{A}_{x}^{M} \mathcal{D}_{x}^{N}\right)^{T}\right] \otimes \mathcal{A}_{y}^{N}=\left[\mathcal{E}_{x}^{R}\left(\mathcal{P}_{x}^{R}\right)^{T}-\mathcal{E}_{x}^{L}\left(\mathcal{P}_{x}^{L}\right)^{T}\right] \otimes \mathcal{A}_{y}^{N} \\
& \mathcal{A}^{P} \mathcal{D}_{y}^{W_{y}}+\left(\mathcal{A}^{W_{y}} \mathcal{D}_{y}^{P}\right)^{T}=\mathcal{A}_{x}^{N} \otimes\left[\mathcal{A}_{y}^{N} \mathcal{D}_{y}^{M}+\left(\mathcal{A}_{y}^{M} \mathcal{D}_{y}^{N}\right)^{T}\right]=\mathcal{A}_{x}^{N} \otimes\left[\mathcal{E}_{y}^{R}\left(\mathcal{P}_{y}^{R}\right)^{T}-\mathcal{E}_{y}^{L}\left(\mathcal{P}_{y}^{L}\right)^{T}\right],
\end{aligned}
$$

which are consequences of the 1D SBP property in equation 13 and the properties of the tensor product operator.

Similarly, the 2D SBP operators appearing in the semi-discretized elastic wave system 19 are constructed from the 1D SBP operators via tensor product as follows

$$
\begin{aligned}
& \mathcal{A}^{V_{x}}=\mathcal{A}_{x}^{M} \otimes \mathcal{A}_{y}^{N}, \mathcal{A}^{\Sigma_{x y}}=\mathcal{A}_{x}^{M} \otimes \mathcal{A}_{y}^{M}, \\
& \mathcal{A}^{V_{y}}=\mathcal{A}_{x}^{N} \otimes \mathcal{A}_{y}^{M}, \mathcal{A}^{\Sigma_{x x}}=\mathcal{A}^{\Sigma_{y y}}=\mathcal{A}_{x}^{N} \otimes \mathcal{A}_{y}^{N} ;
\end{aligned}
$$

and

$$
\begin{aligned}
& \mathcal{D}_{x}^{V_{x}}=\mathcal{D}_{x}^{M} \otimes \mathcal{I}_{y}^{N}, \quad \mathcal{D}_{x}^{V_{y}}=\mathcal{D}_{x}^{N} \otimes \mathcal{I}_{y}^{M}, \quad \mathcal{D}_{x}^{\Sigma_{x y}}=\mathcal{D}_{x}^{M} \otimes \mathcal{I}_{y}^{M}, \quad \mathcal{D}_{x}^{\Sigma_{x x}}=\mathcal{D}_{x}^{N} \otimes \mathcal{I}_{y}^{N}, \\
& \mathcal{D}_{y}^{V_{x}}=\mathcal{I}_{x}^{M} \otimes \mathcal{D}_{y}^{N}, \quad \mathcal{D}_{y}^{V_{y}}=\mathcal{I}_{x}^{N} \otimes \mathcal{D}_{y}^{M}, \mathcal{D}_{y}^{\Sigma_{x y}}=\mathcal{I}_{x}^{M} \otimes \mathcal{D}_{y}^{M}, \quad \mathcal{D}_{y}^{\Sigma_{y y}}=\mathcal{I}_{x}^{N} \otimes \mathcal{D}_{y}^{N} .
\end{aligned}
$$

Constructed as such, these 2D SBP operators satisfy the following relations: 


$$
\begin{aligned}
& \mathcal{A}^{\Sigma_{x x}} \mathcal{D}_{x}^{V_{x}}+\left(\mathcal{A}^{V_{x}} \mathcal{D}_{x}^{\Sigma_{x x}}\right)^{T}=\left[\mathcal{A}_{x}^{N} \mathcal{D}_{x}^{M}+\left(\mathcal{A}_{x}^{M} \mathcal{D}_{x}^{N}\right)^{T}\right] \otimes \mathcal{A}_{y}^{N}=\left[\mathcal{E}_{x}^{R}\left(\mathcal{P}_{x}^{R}\right)^{T}-\mathcal{E}_{x}^{L}\left(\mathcal{P}_{x}^{L}\right)^{T}\right] \otimes \mathcal{A}_{y}^{N} ; \\
& \mathcal{A}^{V_{y}} \mathcal{D}_{x}^{\Sigma_{x y}}+\left(\mathcal{A}^{\Sigma_{x y}} \mathcal{D}_{x}^{V_{y}}\right)^{T}=\left[\mathcal{A}_{x}^{N} \mathcal{D}_{x}^{M}+\left(\mathcal{A}_{x}^{M} \mathcal{D}_{x}^{N}\right)^{T}\right] \otimes \mathcal{A}_{y}^{M}=\left[\mathcal{E}_{x}^{R}\left(\mathcal{P}_{x}^{R}\right)^{T}-\mathcal{E}_{x}^{L}\left(\mathcal{P}_{x}^{L}\right)^{T}\right] \otimes \mathcal{A}_{y}^{M} ; \\
& \mathcal{A}^{V_{x}} \mathcal{D}_{y}^{\Sigma_{x y}}+\left(\mathcal{A}^{\Sigma_{x y}} \mathcal{D}_{y}^{V_{x}}\right)^{T}=\mathcal{A}_{x}^{M} \otimes\left[\mathcal{A}_{y}^{N} \mathcal{D}_{y}^{M}+\left(\mathcal{A}_{y}^{M} \mathcal{D}_{y}^{N}\right)^{T}\right]=\mathcal{A}_{x}^{M} \otimes\left[\mathcal{E}_{y}^{R}\left(\mathcal{P}_{y}^{R}\right)^{T}-\mathcal{E}_{y}^{L}\left(\mathcal{P}_{y}^{L}\right)^{T}\right] ; \\
& \mathcal{A}^{\Sigma_{y y}} \mathcal{D}_{y}^{V_{y}}+\left(\mathcal{A}^{V_{y}} \mathcal{D}_{y}^{\Sigma_{y y}}\right)^{T}=\mathcal{A}_{x}^{N} \otimes\left[\mathcal{A}_{y}^{N} \mathcal{D}_{y}^{M}+\left(\mathcal{A}_{y}^{M} \mathcal{D}_{y}^{N}\right)^{T}\right]=\mathcal{A}_{x}^{N} \otimes\left[\mathcal{E}_{y}^{R}\left(\mathcal{P}_{y}^{R}\right)^{T}-\mathcal{E}_{y}^{L}\left(\mathcal{P}_{y}^{L}\right)^{T}\right],
\end{aligned}
$$

which are again consequences of the 1D SBP property in equation 13 and the properties of the tensor product operator.

\section{APPENDIX D}

\section{ADDITIONAL FIGURES}

This appendix contains two figures $(\bar{D}-1)$ and $\bar{D}-2)$ that are the equivalents of Figures 7 and 8 , with the receiver location placed just outside the right-top corner of the elastic subdomain as illustrated in Figure 6. Specifically, the receiver is placed at one parametric grid spacing (i.e., $20 \mathrm{~m}$ ) to the top and to the right of the top-right corner.

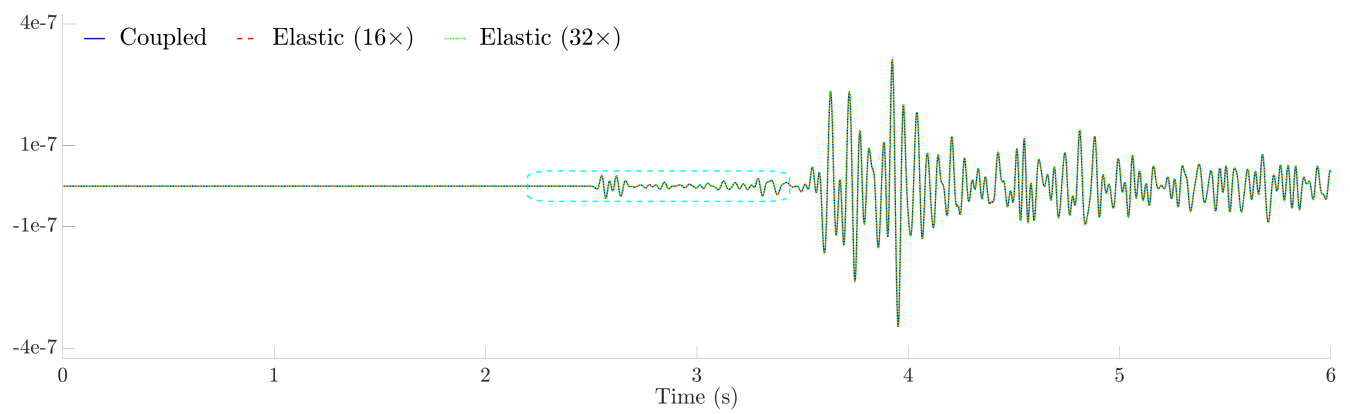

Figure D-1: Time history of $P$ recorded at the receiver location from coupled simulation compared with full elastic simulation results.

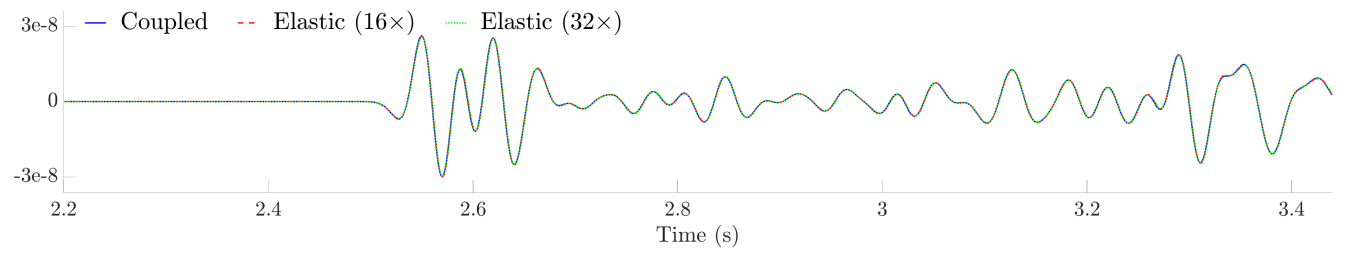

Figure D-2: Zoom-in display of the segment encircled by the dashed lines in Figure D-1.

\section{REFERENCES}

Aminzadeh, F., N. Burkhard, L. Nicoletis, F. Rocca, and K. Wyatt, 1994, SEG/EAEG 3-D modeling project: 2nd update: The Leading Edge, 13, 949-952. 
Appelö, D., and N. A. Petersson, 2009, A stable finite difference method for the elastic wave equation on complex geometries with free surfaces: Communications in Computational Physics, 5, 84-107.

Appelö, D., and S. Wang, 2019, An energy-based discontinuous galerkin method for coupled elasto-acoustic wave equations in second-order form: International Journal for Numerical Methods in Engineering, 119, 618-638.

Banks, J. W., T. Hagstrom, and J. Jacangelo, 2018, Galerkin differences for acoustic and elastic wave equations in two space dimensions: Journal of Computational Physics, 372, 864-892.

Biot, M. A., 1956a, Theory of propagation of elastic waves in a fluid-saturated porous solid. I. Low-frequency range: The Journal of the Acoustical Society of America, 28, 168-178.

- 1956b, Theory of propagation of elastic waves in a fluid-saturated porous solid. II. Higher frequency range: The Journal of the acoustical Society of america, 28, 179-191.

Bourbié, T., O. Coussy, and B. Zinszner, 1987, Acoustics of porous media: Institut Français du Pétrole Publications.

Brown, D. L., 1984, A note on the numerical solution of the wave equation with piecewise smooth coefficients: Mathematics of Computation, 42, 369-391.

Carpenter, M. H., D. Gottlieb, and S. Abarbanel, 1994, Time-stable boundary conditions for finite-difference schemes solving hyperbolic systems: methodology and application to high-order compact schemes: Journal of Computational Physics, 111, 220-236.

Castillo, J. E., and G. F. Miranda, 2013, Mimetic discretization methods: CRC Press.

Chaljub, E., Y. Capdeville, and J.-P. Vilotte, 2003, Solving elastodynamics in a fluid-solid heterogeneous sphere: a parallel spectral element approximation on non-conforming grids: Journal of Computational Physics, 187, 457-491.

de la Puente, J., M. Ferrer, M. Hanzich, J. E. Castillo, and J. M. Cela, 2014, Mimetic seismic wave modeling including topography on deformed staggered grids: Geophysics, 79, no. 3, T125-T141.

Del Rey Fernández, D. C., J. E. Hicken, and D. W. Zingg, 2014, Review of summationby-parts operators with simultaneous approximation terms for the numerical solution of partial differential equations: Computers \& Fluids, 95, 171-196.

Gao, L., D. C. Del Rey Fernández, M. Carpenter, and D. Keyes, 2019, SBP-SAT finite difference discretization of acoustic wave equations on staggered block-wise uniform grids: Journal of Computational and Applied Mathematics, 348, 421-444.

Gao, L., and D. Keyes, 2019, Combining finite element and finite difference methods for isotropic elastic wave simulations in an energy-conserving manner: Journal of Computational Physics, 378, 665-685.

Gregory, A., 1976, Fluid saturation effects on dynamic elastic properties of sedimentary rocks: Geophysics, 41, 895-921.

Hamilton, E. L., 1979, Vp/Vs and Poisson's ratios in marine sediments and rocks: The Journal of the Acoustical Society of America, 66, 1093-1101.

Hicken, J. E., and D. W. Zingg, 2013, Summation-by-parts operators and high-order quadrature: Journal of Computational and Applied Mathematics, 237, 111-125.

Jackson, M. P., and M. R. Hudec, 2017, Salt tectonics: Principles and practice: Cambridge University Press.

Jones, I. F., and I. Davison, 2014, Seismic imaging in and around salt bodies: Interpretation, 2, no. 4, SL1-SL20.

Käser, M., and M. Dumbser, 2008, A highly accurate discontinuous Galerkin method for complex interfaces between solids and moving fluids: Geophysics, 73, no. 3, T23-T35. 
Komatitsch, D., C. Barnes, and J. Tromp, 2000, Wave propagation near a fluid-solid interface: A spectral-element approach: Geophysics, 65, 623-631.

Kozdon, J. E., and E. M. Dunham, 2014, Constraining shallow slip and tsunami excitation in megathrust ruptures using seismic and ocean acoustic waves recorded on ocean-bottom sensor networks: Earth and Planetary Science Letters, 396, 56-65.

Kreiss, H.-O., and G. Scherer, 1974, Finite element and finite difference methods for hyperbolic partial differential equations. in: Mathematical aspects of finite elements in partial differential equations: Academic Press.

Levander, A. R., 1988, Fourth-order finite-difference P-SV seismograms: Geophysics, 53, $1425-1436$.

Leveille, J. P., I. F. Jones, Z.-Z. Zhou, B. Wang, and F. Liu, 2011, Subsalt imaging for exploration, production, and development: A review: Geophysics, 76, no. 5, WB3-WB20.

Lotto, G. C., and E. M. Dunham, 2015, High-order finite difference modeling of tsunami generation in a compressible ocean from offshore earthquakes: Computational Geosciences, 19, 327-340.

Lu, R. S., D. E. Willen, and I. A. Watson, 2003, Identifying, removing, and imaging PS conversions at salt-sediment interfaces: Geophysics, 68, 1052-1059.

Madariaga, R., 1976, Dynamics of an expanding circular fault: Bulletin of the Seismological Society of America, 66, 639-666.

Mattsson, K., and M. H. Carpenter, 2010, Stable and accurate interpolation operators for high-order multiblock finite difference methods: SIAM Journal on Scientific Computing, 32, 2298-2320.

Michler, C., L. Demkowicz, and C. Torres-Verdín, 2009, Numerical simulation of borehole acoustic logging in the frequency and time domains with hp-adaptive finite elements: Computer Methods in Applied Mechanics and Engineering, 198, 1821-1838.

Ogilvie, J. S., and G. W. Purnell, 1996, Effects of salt-related mode conversions on subsalt prospecting: Geophysics, 61, 331-348.

O'Reilly, O., T. Lundquist, E. M. Dunham, and J. Nordström, 2017, Energy stable and highorder-accurate finite difference methods on staggered grids: Journal of Computational Physics, 346, 572-589.

O'Reilly, O., and N. A. Petersson, 2020, Energy conservative SBP discretizations of the acoustic wave equation in covariant form on staggered curvilinear grids: Journal of Computational Physics, 109386.

Petersson, N. A., and B. Sjögreen, 2015, Wave propagation in anisotropic elastic materials and curvilinear coordinates using a summation-by-parts finite difference method: Journal of Computational Physics, 299, 820-841.

,- 2018 , High order accurate finite difference modeling of seismo-acoustic wave propagation in a moving atmosphere and a heterogeneous earth model coupled across a realistic topography: Journal of Scientific Computing, 74, 290-323.

Qu, Y., Z. Guan, J. Li, and Z. Li, 2020, Fluid-solid coupled full-waveform inversion in the curvilinear coordinates for ocean-bottom cable data: Geophysics, 85, no. 3, R113-R133.

Rodríguez-Rozas, Á., and J. Diaz, 2016, Non-conforming curved finite element schemes for time-dependent elastic-acoustic coupled problems: Journal of Computational Physics, 305, 44-62.

Rojas, O., S. Day, J. Castillo, and L. A. Dalguer, 2008, Modelling of rupture propagation using high-order mimetic finite differences: Geophysical Journal International, 172, 631650 .

Schuster, G. T., 2014, Seismic imaging, overview. in: Gupta H.K. (eds) Encyclopedia of 
Solid Earth Geophysics: Springer Netherlands.

Shragge, J., and B. Tapley, 2017, Solving the tensorial 3D acoustic wave equation: A mimetic finite-difference time-domain approach: Geophysics, 82, no. 4, T183-T196.

Singh, H., J. Shragge, and I. Tsvankin, 2019, Coupled-domain acoustic-elastic solver for anisotropic media: A mimetic finite difference approach, in SEG Technical Program Expanded Abstracts 2019: Society of Exploration Geophysicists, 3755-3759.

Stein, S., and M. Wysession, 2009, An introduction to seismology, earthquakes, and earth structure: John Wiley \& Sons.

Stephen, R., 1983, A comparison of finite difference and reflectivity seismograms for marine models: Geophysical Journal International, 72, 39-57.

Stephen, R. A., F. Cardo-Casas, and C. Cheng, 1985, Finite-difference synthetic acoustic logs: Geophysics, 50, 1588-1609.

Svärd, M., and J. Nordström, 2014, Review of summation-by-parts schemes for initialboundary-value problems: Journal of Computational Physics, 268, 17-38.

Symes, W. W., 2009, The seismic reflection inverse problem: Inverse problems, 25, 123008.

Symes, W. W., and T. Vdovina, 2009, Interface error analysis for numerical wave propagation: Computational Geosciences, 13, 363-371.

Terrana, S., J. Vilotte, and L. Guillot, 2018, A spectral hybridizable discontinuous Galerkin method for elastic-acoustic wave propagation: Geophysical Journal International, 213, 574-602.

Virieux, J., 1984, SH-wave propagation in heterogeneous media: Velocity-stress finitedifference method: Geophysics, 49, 1933-1942.

— 1986 , P-SV wave propagation in heterogeneous media: Velocity-stress finitedifference method: Geophysics, 51, 889-901.

Virieux, J., and S. Operto, 2009, An overview of full-waveform inversion in exploration geophysics: Geophysics, 74, no. 6, WCC1-WCC26.

Wang, S., K. Virta, and G. Kreiss, 2016, High order finite difference methods for the wave equation with non-conforming grid interfaces: Journal of Scientific Computing, 68, 10021028.

Wilcox, L. C., G. Stadler, C. Burstedde, and O. Ghattas, 2010, A high-order discontinuous Galerkin method for wave propagation through coupled elastic-acoustic media: Journal of Computational Physics, 229, 9373-9396.

Ye, R., M. V. de Hoop, C. L. Petrovitch, L. J. Pyrak-Nolte, and L. C. Wilcox, 2016, A discontinuous Galerkin method with a modified penalty flux for the propagation and scattering of acousto-elastic waves: Geophysical Journal International, 205, 1267-1289.

Yee, K., 1966, Numerical solution of initial boundary value problems involving Maxwell's equations in isotropic media: IEEE Transactions on antennas and propagation, 14, 302307. 